\title{
Numerical simulation of surface waves instability on a homogeneous grid
}

\author{
Alexander O. Korotkevicha,b,*, Alexander I. Dyachenko ${ }^{\mathrm{b}, \mathrm{e}}$, Vladimir E. \\ Zakharov ${ }^{\mathrm{c}, \mathrm{d}, \mathrm{e}, \mathrm{f}, \mathrm{b}}$ \\ ${ }^{a}$ Department of Mathematics $\mathcal{E}$ Statistics, The University of New Mexico, MSC01 1115, 1 \\ University of New Mexico, Albuquerque, New Mexico, 87131-0001, USA \\ ${ }^{b}$ L. D. Landau Institute for Theoretical Physics, 2 Kosygin Str., Moscow, 119334, Russian \\ Federation \\ ${ }^{c}$ University of Arizona, Department of Mathematics, Tucson, AZ 85721, USA \\ ${ }^{d}$ P. N. Lebedev Physical Institute, 53 Leninskiy prospekt, Moscow, 119334, Russian Federation \\ ${ }^{e}$ Laboratory of Nonlinear Wave Processes, Novosibirsk State University, Novosibirsk, Russian \\ Federation \\ ${ }^{f}$ Waves and Solitons LLC,738 W. Sereno Dr., Gilbert, AZ, USA
}

\begin{abstract}
We performed full-scale numerical simulation of instability of weakly nonlinear waves on the surface of deep fluid. We show that the instability development leads to chaotization and formation of wave turbulence.

Instability of both propagating and standing waves were studied. We separately studied pure capillary wave, that was unstable due to three-wave interactions and pure gravity waves, that were unstable due to four-wave interactions. The theoretical description of instabilities in all cases is included in the article. The numerical algorithm used in these and many other previous simulations performed by the authors is described in detail.
\end{abstract}

Keywords:

PACS:

\section{Introduction.}

Stationary propagating waves on the surface of deep, heavy ideal fluid have been known since the middle of the nineteenth century. Stokes (see, for instance [1]) in 1847 found the solution of the Euler equation in the form of

${ }^{*}$ Corresponding author

Email address: alexkor@math.unm.edu (Alexander O. Korotkevich) 
trigonometric series. For the shape of surface $\eta(x, t)$, he obtained:

$$
\eta(x, t)=a\left[\cos (k x-\omega t)+\frac{1}{2} \mu \cos \{2(k x-\omega t)\}+\frac{3}{8} \mu^{2} \cos \{3(k x-\omega t)\}+\ldots\right] .
$$

Here we introduced steepness $\mu$ and frequency $\omega$

$$
\mu=k a, \quad \omega=\sqrt{g k}\left(1+\frac{1}{2} \mu^{2}+\frac{1}{8} \mu^{4}+\ldots\right) .
$$

Stokes found two algorithms for the calculation of all terms in series (1) and (2) (see Sretenskii [2]). Convergence of these series was proven by Nekrasov [3, 4] in 1921. Another proof was found by Levi-Chivita [5]. Recently shapes of different Stokes waves were obtained numerically with high precision [6, 7], their analytic structure was revealed [7] and explained [8].

It has been known since 1965 [9] that stationary waves on the surface of deep water are unstable. The theory of instability [10, 11, 12, 13] was developed for waves of small amplitude within the limit $\mu \rightarrow 0$. A history of this question is described in the article [14]. Recent advances can be found in [15]. In the present paper, we study the instability of stationary waves numerically through the direct solution of the Euler equation which describes a potential flow of ideal fluid with free surface. This approach has two important advantages. Firstly, through numerical simulation we can study waves with finite amplitudes. While this paper focuses only on cases of small amplitude, such and advantage will be crucial for other applications, e.g. wave breaking simulation. Secondly, the use of numerical simulation allows us to study not only linear, but also nonlinear stages of instability development. Even in integrable systems like the NLSE, analytical study of the monochromatic wave is a very nonlinear problem and can be solved only by methods of algebraic geometry [16]. In more realistic models, development of a nonlinear theory of modulational instability for waves is a hopeless problem. In the long run, we have to expect that instability will lead to the formation of a stochastic wave field described by a kinetic equation for squared wave amplitudes and formation of Kolmogorov-Zakharov (KZ) spectra, governed by the energy flux in high wave numbers [17].

The article is organized as follows. Chapters 2 and 3 are devoted to analytical theory of stability of weakly nonlinear stationary waves. To develop this theory, we use Hamiltonian formalism as this approach is the most compact and suitable. We start with presenting the Euler equation of ideal fluid with free surface in the Hamiltonian form. Surface tension is also included in the Hamiltonian. In the presence of surface tension, the dispersion relation is:

$$
\omega_{k}=\underset{2}{\sqrt{g k+\sigma k^{3}}},
$$


where $\sigma$ - the surface tension coefficient (here and further we consider fluid of unit density).

Wave vectors of small-amplitude stationary waves are solutions of the equation

$$
\omega_{k}=c k
$$

This equation has two solutions (we omit trivial solution $k=0$ ):

$$
k_{1,2}=\frac{c^{2} \pm \sqrt{c^{4}-4 g \sigma}}{2 \sigma}
$$

if $c>c_{0}$, where $c_{0}=(4 g \sigma)^{1 / 4}$. For water, $c_{0} \simeq 23 \mathrm{~cm} / \mathrm{sec}$. In a generic case $c \sim$ $c_{0}$ stationary waves comprise a complicated four-parameter family. However, in the limiting case $c \gg c_{0}$ one can split it in two periodic families of "pure gravitational" and "pure capillary" waves.

The Stokes wave is "pure gravitational". Now, with $k_{1}=g / c^{2}$ capillary effects can be neglected. In the "pure capillary" case $k_{2}=c^{2} / \sigma$, effects of gravity can be ignored. All stationary waves on the surface of deep fluid are unstable. However, the instabilities of short capillary waves and long gravity waves are significantly different and described by different "efficient Hamiltonians". The case of "pure capillary" waves is the simplest. The instability can be studied if Hamiltonian contains only quadratic and cubic terms. This is the subject of Chapter 2. A situation is more complicated for gravitational waves. In this case, fourth order terms must be included in the Hamiltonian. Then, one has to exclude the cubic terms through a proper conformal transformation. As a result, we get so-called "Zakharov equation" [13]. In the framework of this equation, the problem of the Stokes wave stability can be solved exactly. This is the subject of Chapter 3.

In Chapter 4, we give a detailed description of the numerical code which we used for the solution of the Hamiltonian Euler equation. This code was used in many papers but was never described in detail [18, 19, 20, 21, 22, 23, 24, 25]. We should stress that in our numerical experiments we worked with the Euler equation written in "natural variables". These equations are not as good for the direct analytical study as they are good for the implementation of numerical method. The structure of nonlinear parts of the Hamiltonian in "natural variables" is relatively simple, and numerical implementation through standard Fast Fourier Transform (FFT) is quite feasible.

In Chapter 5 we present our results on the modeling of capillary wave instability. We show that an initial stage of instability is described pretty well by 
the linear analytical theory. Further development of instability leads to the appearance of "secondary instabilities" and a tendency toward the formation of a chaotic wave field, which should be described by statistical methods.

In Chapter 6, we study the instability of the Stokes wave. We show that this instability is mostly "modulational". In other words, the wave remains quasimonochromatic for a long time after the development of the instability.

Finally, in Chapter 7 we present first results on the development of the standing wave instability. We show that this instability leads to fast isotropization of the wave field. This mechanism can be used in experiments for generation of an isotropic wave field.

\section{Theory of decay instability}

In this section, we develop the simplest version of the theory of stationary wave instability. This simple theory is applicable if triple-wave nonlinear processes governed by the resonant conditions

$$
\begin{array}{r}
\omega_{k}=\omega_{k_{1}}+\omega_{k_{2}}, \\
\mathbf{k}=\mathbf{k}_{1}+\mathbf{k}_{2} .
\end{array}
$$

are permitted. Let us briefly describe how the theory of surface waves can be embedded into the general Hamiltonian theory of nonlinear waves, before we use conditions (5).

Suppose that ideal incompressible fluid fills the space $-\infty<z<\eta(\mathbf{r}, t)$, here $\mathbf{r}=(x, y)-$ two dimensional vector. A flow is potential $\mathbf{v}=\nabla \Phi$, hence hydrodynamical potential $\Phi$ satisfies the Laplace equation

$$
\nabla^{2} \Phi=0
$$

Let us define $\psi=\left.\Phi\right|_{z=n}$ and impose a natural boundary condition $\Phi_{z} \rightarrow 0$ at $z \rightarrow-\infty$. It is known [10] that $\eta(\mathbf{r}, t)$ and $\psi(\mathbf{r}, t)$ are canonically conjugated variables satisfying evolutionary equations

$$
\frac{\partial \eta}{\partial t}=\frac{\delta H}{\delta \psi}, \quad \frac{\partial \psi}{\partial t}=-\frac{\delta H}{\delta \eta}
$$

Here $H=T+U-$ total energy of the fluid, consisting of kinetic energy

$$
T=\frac{1}{2} \int \mathrm{d}^{2} \mathbf{r} \int_{-\infty}^{\eta}(\nabla \Phi)^{2} \mathrm{~d} z
$$


and potential energy

$$
U=\frac{g}{2} \int \eta^{2} \mathrm{~d}^{2} \mathbf{r}+\sigma \int\left(\sqrt{1+(\nabla \eta)^{2}}-1\right) \mathrm{d}^{2} \mathbf{r} .
$$

The Hamiltonian $H$ in terms of $\eta$ and $\psi$ is given by the infinite series

$$
H=H_{0}+H_{1}+H_{2}+\ldots
$$

Here

$$
\begin{array}{r}
H_{0}=\frac{1}{2} \int\left\{\psi \hat{k} \psi+g \eta^{2}+\sigma(\nabla \eta)^{2}\right\} \mathrm{d}^{2} \mathbf{r}, \\
\text { here } \hat{k} \psi=\sqrt{-\nabla^{2}} \psi, \\
H_{1}=\frac{1}{2} \int \eta\left\{|\nabla \psi|^{2}-(\hat{k} \psi)^{2}\right\} \mathrm{d}^{2} \mathbf{r}, \\
H_{2}=\frac{1}{2} \int \eta(\hat{k} \psi)\left[\hat{k}(\eta \hat{k} \psi)+\eta \nabla^{2} \psi\right] \mathrm{d}^{2} \mathbf{r}+\frac{1}{2} \sigma \int\left(\nabla \eta^{2}\right)^{2} \mathrm{~d}^{2} \mathbf{r} .
\end{array}
$$

Thereafter, we will neglect the last term in (13).

One can perform the symmetric Fourier transform

$$
\psi_{\mathbf{k}}=\frac{1}{2 \pi} \int \psi(\mathbf{r}) \mathrm{e}^{-l \mathbf{k r}} \mathrm{d}^{2} \mathbf{r}, \quad \eta_{\mathbf{k}}=\frac{1}{2 \pi} \int \eta(\mathbf{r}) \mathrm{e}^{-l \mathbf{k r}} \mathrm{d}^{2} \mathbf{r} .
$$

This is the canonical transformation. Equations (7) now take the form

$$
\frac{\partial \eta}{\partial t}=\frac{\delta H}{\delta \psi^{*}}, \quad \frac{\partial \psi}{\partial t}=-\frac{\delta H}{\delta \eta^{*}}
$$

Now

$$
\begin{aligned}
& H_{0}=\frac{1}{2} \int\left(|k|\left|\psi_{\mathbf{k}}\right|^{2}+\sigma|k|^{2}\left|\eta_{\mathbf{k}}\right|^{2}+g\left|\eta_{\mathbf{k}}\right|^{2}\right) \mathrm{d} \mathbf{k}, \\
& H_{1}=-\frac{1}{4 \pi} \int L_{\mathbf{k}_{1} \mathbf{k}_{2}} \psi_{\mathbf{k}_{1}} \psi_{\mathbf{k}_{2}} \eta_{\mathbf{k}_{3}} \delta\left(\mathbf{k}_{1}+\mathbf{k}_{2}+\mathbf{k}_{3}\right) \mathrm{d} \mathbf{k}_{1} \mathrm{~d} \mathbf{k}_{2} \mathrm{~d} \mathbf{k}_{3}, \\
& H_{2}=\frac{1}{16 \pi^{2}} \int M_{\mathbf{k}_{1} \mathbf{k}_{2} \mathbf{k}_{3} \mathbf{k}_{4}} \psi_{\mathbf{k}_{1}} \psi_{\mathbf{k}_{2}} \eta_{\mathbf{k}_{3}} \eta_{\mathbf{k}_{4}} \delta\left(\mathbf{k}_{1}+\mathbf{k}_{2}+\mathbf{k}_{3}+\mathbf{k}_{4}\right) \mathrm{d} \mathbf{k}_{1} \mathrm{~d}_{2} \mathbf{d}_{\mathbf{k}_{3}} \mathrm{~d} \mathbf{k}_{4},
\end{aligned}
$$

Here

$$
\begin{aligned}
& L_{\mathbf{k}_{1} \mathbf{k}_{2}}=\left(\mathbf{k}_{1} \mathbf{k}_{2}\right)+\left|\mathbf{k}_{1}\right|\left|\mathbf{k}_{2}\right| \\
& M_{\mathbf{k}_{1} \mathbf{k}_{2} \mathbf{k}_{3} \mathbf{k}_{4}}=\left|\mathbf{k}_{1}\right|\left|\mathbf{k}_{2}\right|\left[\frac { 1 } { 2 } \left(\left|\mathbf{k}_{1}+\mathbf{k}_{3}\right|+\left|\mathbf{k}_{1}+\mathbf{k}_{4}\right|+\right.\right. \\
& \left.\left.+\left|\mathbf{k}_{3}+\mathbf{k}_{2}\right|+\left|\mathbf{k}_{2}+\mathbf{k}_{4}\right|\right)-\left|\mathbf{k}_{1}\right|-\underset{5}{\mid}\left|\mathbf{k}_{2}\right|\right] .
\end{aligned}
$$


Equations (7) written for the Hamiltonian (12,13) read

$$
\begin{aligned}
\dot{\eta}= & \hat{k} \psi-(\nabla(\eta \nabla \psi))-\hat{k}[\eta \hat{k} \psi]+\hat{k}(\eta \hat{k}[\eta \hat{k} \psi])+ \\
& +\frac{1}{2} \nabla^{2}\left[\eta^{2} \hat{k} \psi\right]+\frac{1}{2} \hat{k}\left[\eta^{2} \nabla^{2} \psi\right], \\
\dot{\psi}= & \sigma \nabla^{2} \eta-g \eta-\frac{1}{2}\left[(\nabla \psi)^{2}-(\hat{k} \psi)^{2}\right]-[\hat{k} \psi] \hat{k}[\eta \hat{k} \psi]-[\eta \hat{k} \psi] \nabla^{2} \psi .
\end{aligned}
$$

These equations were considered for the first time in [26]. Equations (18) are basic in our numerical simulations. To develop an analytical theory of stationary wave instability, we use equation (15). It is interesting to note that even for capillary waves in some cases, it is worthwhile to keep cubic terms in (18) as it is shown in Appendix C.

Let us introduce the complex normal variables

$$
a_{\mathbf{k}}=\sqrt{\frac{\omega_{k}}{2 k}} \eta_{\mathbf{k}}+\imath \sqrt{\frac{k}{2 \omega_{k}}} \psi_{\mathbf{k}} .
$$

As far as $\eta_{-\mathbf{k}}=\eta_{\mathbf{k}}^{*}, \psi_{-\mathbf{k}}=\psi_{\mathbf{k}}^{*}$ (because these are Fourier transforms of real functions), we have

$$
\eta_{\mathbf{k}}=\sqrt{\frac{2 k}{\omega_{k}}}\left(a_{\mathbf{k}}+a_{-\mathbf{k}}^{*}\right), \quad \psi_{\mathbf{k}}=-l \sqrt{\frac{2 \omega_{k}}{k}}\left(a_{\mathbf{k}}-a_{-\mathbf{k}}^{*}\right) .
$$

In terms of $a_{\mathbf{k}}$, equations (15) turn into one equation

$$
\frac{\partial a_{\mathbf{k}}}{\partial t}=-l \frac{\delta H}{\delta a_{\mathbf{k}}^{*}}
$$

Now

$$
H_{0}=\int \omega_{k}\left|a_{\mathbf{k}}\right|^{2} \mathrm{~d}^{2} \mathbf{k}
$$

Then

$$
H_{1}=H_{1}^{(0,3)}+H_{1}^{(1,2)} .
$$

Here

$$
\begin{aligned}
& H_{1}^{(0,3)}=\frac{1}{6} \int V_{\mathbf{k k}_{1} \mathbf{k}_{2}}^{(0,3)}\left(a_{\mathbf{k}} a_{\mathbf{k}_{1}} a_{\mathbf{k}_{2}}+a_{\mathbf{k}}^{*} a_{\mathbf{k}_{1}}^{*} a_{\mathbf{k}_{2}}^{*}\right) \delta\left(\mathbf{k}+\mathbf{k}_{1}+\mathbf{k}_{2}\right) \mathrm{d} \mathbf{k} \mathrm{d} \mathbf{k}_{1} \mathrm{~d} \mathbf{k}_{2} \\
& H_{1}^{(1,2)}=\frac{1}{2} \int V_{\mathbf{k} \mathbf{k}_{1} \mathbf{k}_{2}}^{(1,2)}\left(a_{\mathbf{k}}^{*} a_{\mathbf{k}_{1}} a_{\mathbf{k}_{2}}+a_{\mathbf{k}} a_{\mathbf{k}_{1}}^{*} a_{\mathbf{k}_{2}}^{*}\right) \delta\left(\mathbf{k}-\mathbf{k}_{1}-\mathbf{k}_{2}\right) \mathrm{d} \mathbf{k} \mathrm{d} \mathbf{k}_{1} \mathrm{~d} \mathbf{k}_{2} .
\end{aligned}
$$


In a similar way

$$
H_{2}=H_{2}^{(0,4)}+H_{2}^{(1,3)}+H_{2}^{(2,2)}
$$

Only the last term in $\mathrm{H}_{2}$ is important for us

$$
H_{2}^{(2,2)}=\frac{1}{4} \int V_{\mathbf{k k}_{1} \mathbf{k}_{2} \mathbf{k}_{3}}^{(2,2)} a_{\mathbf{k}} a_{\mathbf{k}_{1}} a_{\mathbf{k}_{2}}^{*} a_{\mathbf{k}_{3}}^{*} \delta\left(\mathbf{k}+\mathbf{k}_{1}-\mathbf{k}_{2}-\mathbf{k}_{3}\right) \mathrm{d} \mathbf{k} \mathrm{d} \mathbf{k}_{1} \mathrm{~d} \mathbf{k}_{2} \mathrm{~d} \mathbf{k}_{3} .
$$

Explicit expressions for $V_{\mathbf{k k}_{1} \mathbf{k}_{2}}^{(0,3)}, V_{\mathbf{k k}_{1} \mathbf{k}_{2}}^{(1,2)}$, and $V_{\mathbf{k k}_{1} \mathbf{k}_{2} \mathbf{k}_{3}}^{(2,2)}$ are presented in Appendix B.

Now everything depends on the shape of function $\omega(k)$. If resonant conditions (5) have real solutions, one can neglect $H_{2}$ and even $H_{3}^{(0,3)}$. Now equations (21) takes a simple form

$$
\begin{aligned}
& \dot{a}_{\mathbf{k}}+\imath \omega_{k} a_{\mathbf{k}}= \\
& -\frac{l}{2} \int\left\{V_{\mathbf{k}_{1} \mathbf{k}_{2}}^{(1,2)} a_{\mathbf{k}_{1}} a_{\mathbf{k}_{2}} \delta\left(\mathbf{k}-\mathbf{k}_{1}-\mathbf{k}_{2}\right)+2 V_{\mathbf{k}_{1} \mathbf{k k}_{2}}^{(1,2)} a_{\mathbf{k}_{1}} a_{\mathbf{k}_{2}}^{*} \delta\left(\mathbf{k}-\mathbf{k}_{1}+\mathbf{k}_{2}\right)\right\} \mathrm{d} \mathbf{k}_{1} \mathrm{~d} \mathbf{k}_{2}
\end{aligned}
$$

Thereafter, we assume

$$
V_{\mathbf{0}, \mathbf{k},-\mathbf{k}}^{(1,2)}=0 .
$$

For surface waves, this condition is satisfied.

Equation (28) has a solution which can be treated as a stationary wave

$$
a_{\mathbf{k}}=\sum_{n=1}^{\infty}\left[a_{n} \mathrm{e}^{-l n \Omega t} \delta\left(\mathbf{k}-n \mathbf{k}_{0}\right)+b_{n} \mathrm{e}^{i n \Omega t} \delta\left(\mathbf{k}+n \mathbf{k}_{0}\right)\right] .
$$

We put $a_{1}=\varepsilon$. Here $\mathbf{k}_{0}$ - an arbitrary wave vector. Coefficients $a_{n}$ are presented by power series

$$
a_{n}=\varepsilon^{n}\left(a_{n}^{(0)}+\varepsilon a_{n}^{(1)}+\ldots\right)
$$

while coefficients $b_{n}$ look as follows:

$$
b_{n}=\varepsilon^{n+2}\left(b_{n}^{(0)}+\varepsilon b_{n}^{(1)}+\ldots\right) .
$$

The frequency of the stationary wave is presented by a series in even powers of $\varepsilon$

$$
\Omega=\omega\left(k_{0}\right)+\varepsilon^{2} \Delta_{1}+\varepsilon^{4} \Delta_{2}+\ldots
$$

Now, let us suppose $\varepsilon \rightarrow 0$. From (31), we see that the first nonlinear correction to frequency is proportional to $\varepsilon^{2}$. Now we will show that the solution (30) is 
unstable, and the growth rate of instability is proportional to $\varepsilon$. It means that all nonlinear corrections to $a_{n}, b_{n}$, and $\Delta_{n}$ can be neglected, and one should look for a solution in the following form:

$$
a_{\mathbf{k}}=\varepsilon \mathrm{e}^{-l \omega\left(k_{0}\right) t} \delta\left(\mathbf{k}-\mathbf{k}_{0}\right)+\alpha(t) \mathrm{e}^{-l \omega\left(\kappa_{1}\right) t} \delta\left(\mathbf{k}-\kappa_{1}\right)+\beta(t) \mathrm{e}^{-\imath \omega\left(\kappa_{2}\right) t} \delta\left(\mathbf{k}-\kappa_{2}\right),
$$

where $\kappa_{1}+\kappa_{2}=\mathbf{k}_{0}$. Then, we linearize the equation (28) and find that $\alpha$ and $\beta$ obey the system of ordinary differential equations

$$
\begin{array}{ll}
\dot{\alpha}=\imath \mathrm{e}^{-\imath \Delta} \varepsilon V \beta^{*}, & V=V_{\mathbf{k}_{0} \kappa_{1} \kappa_{2}}^{(1,2)}, \\
\dot{\beta}=\imath \mathrm{e}^{-\imath \Delta} \varepsilon V \alpha^{*}, & \Delta=\omega\left(k_{0}\right)-\omega\left(\kappa_{1}\right)-\omega\left(\kappa_{2}\right) .
\end{array}
$$

A general solution of equation (33) is

$$
\alpha=\alpha_{0} \mathrm{e}^{(-l \Delta / 2 \pm \gamma) t}, \quad \beta=\beta_{0} \mathrm{e}^{(-l \Delta / 2 \pm \gamma) t} .
$$

Here

$$
\gamma=\sqrt{\varepsilon^{2}|V|^{2}-\frac{1}{4} \Delta^{2}}
$$

and $\alpha_{0}, \beta_{0}$ are connected by relation

$$
\left(-\frac{\imath \Delta}{2} \pm \gamma\right) \beta_{0}=\imath \varepsilon V \alpha_{0}^{*} .
$$

Instability takes place if wave vectors $\kappa_{1}, \kappa_{2}$ are posed near the surface (or the curve)

$$
\begin{aligned}
& \omega\left(k_{0}\right)=\omega\left(\kappa_{1}\right)+\omega\left(\kappa_{2}\right), \\
& \mathbf{k}_{0}=\kappa_{1}+\kappa_{2} .
\end{aligned}
$$

The maximum of the growth rate $\gamma=\varepsilon|V|$ is reached exactly on the resonant surface, which is represented in Figure 11. The plot of growth rate (35) on a homogeneous grid of wave numbers for $2 \pi \times 2 \pi$ periodic box is given in Figure 2 .

\section{Four-waves instability.}

The theory developed in the previous chapter is applicable to the study of capillary wave instability. In the case of gravity waves, resonant conditions (37) 


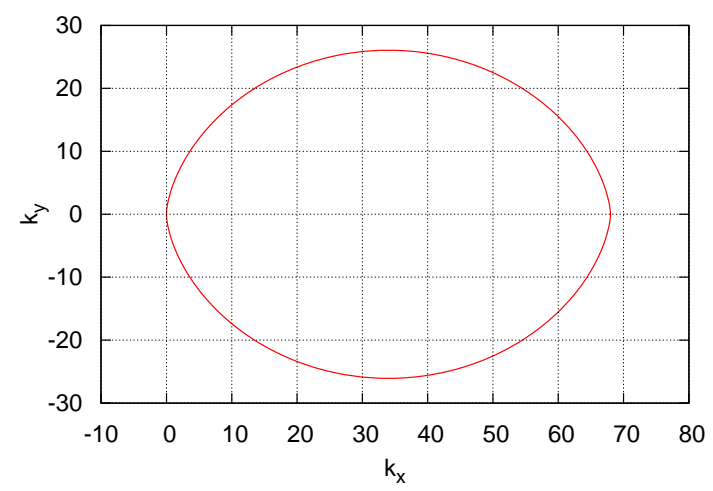

Figure 1: Resonant curve for decay of monochromatic capillary wave with $\mathbf{k}=(68,0)$.
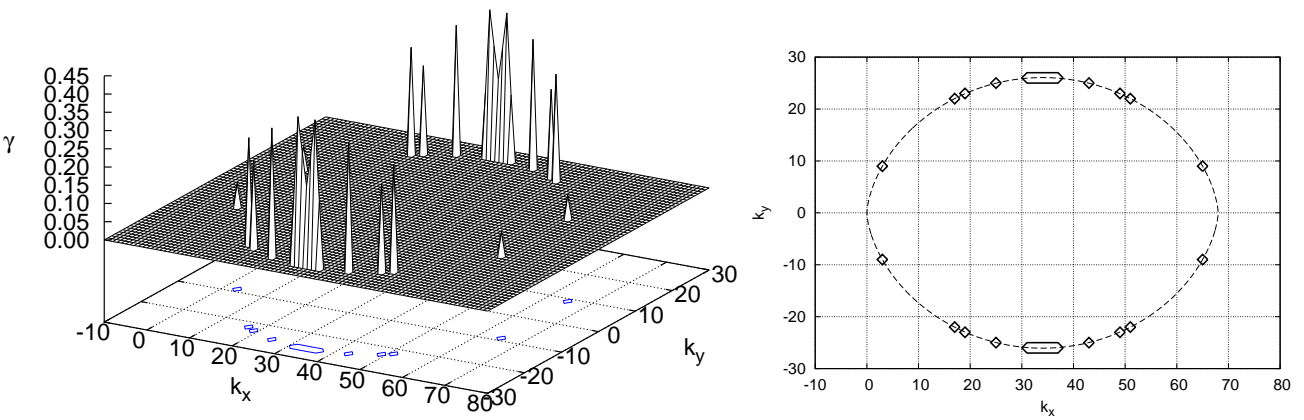

Figure 2: Growth rate for decay instability of monochromatic capillary wave with $\mathbf{k}_{0}=(68,0)$ and average steepness $\mu=0.05$ on the homogeneous grid. Left panel: isometric projection; right panel: contour of the surface at the level $10^{-23}$; dashed line - resonance curve. 
have no real solutions. It means that cubic terms in the Hamiltonian can be excluded by a proper canonical transformation given by power series [27, 28]

$$
\begin{aligned}
& a_{\mathbf{k}}=b_{\mathbf{k}}^{(0)}+b_{\mathbf{k}}^{(1)}+b_{\mathbf{k}}^{(2)}+\ldots \\
& b_{\mathbf{k}}^{(0)}=a_{\mathbf{k}} .
\end{aligned}
$$

Next terms $b_{\mathbf{k}}^{(1)}, b_{\mathbf{k}}^{(2)}$ are presented in Appendix B.

After canonical transformation, the Hamiltonian is reduced to the form

$$
\begin{array}{r}
H=H_{0}+\tilde{H}_{2}, \\
\tilde{H}_{2}=\frac{1}{4} \int T_{\mathbf{k k}_{1} \mathbf{k}_{2} \mathbf{k}_{3}} b_{\mathbf{k}}^{*} b_{\mathbf{k}_{1}}^{*} b_{\mathbf{k}_{2}} b_{\mathbf{k}_{3}} \delta\left(\mathbf{k}+\mathbf{k}_{1}-\mathbf{k}_{2}-\mathbf{k}_{3}\right) \mathrm{d} \mathbf{k} \mathrm{d} \mathbf{k}_{1} \mathrm{~d} \mathbf{k}_{2} \mathrm{~d} \mathbf{k}_{3} .
\end{array}
$$

The explicit and complicated expression for $T_{\mathbf{k k}_{1} \mathbf{k}_{2} \mathbf{k}_{3}}$ is given in Appendix B. $T_{\mathbf{k k}_{1} \mathbf{k}_{2} \mathbf{k}_{3}}$ is a homogeneous function of the third order.

Now, canonical variable $b_{\mathbf{k}}$ obeys the so called "Zakharov's equation" [13]

$$
\dot{b}_{\mathbf{k}}+\imath \omega_{k} b_{\mathbf{k}}=-\frac{l}{2} \int T_{\mathbf{k k}_{1} \mathbf{k}_{2} \mathbf{k}_{3}} b_{\mathbf{k}_{1}}^{*} b_{\mathbf{k}_{2}} b_{\mathbf{k}_{3}} \delta\left(\mathbf{k}+\mathbf{k}_{1}-\mathbf{k}_{2}-\mathbf{k}_{3}\right) \mathrm{d} \mathbf{k}_{1} \mathrm{~d} \mathbf{k}_{2} \mathrm{~d} \mathbf{k}_{3}
$$

Equation (41) has the exact solution

$$
\begin{gathered}
b_{\mathbf{k}}=A \delta\left(\mathbf{k}-\mathbf{k}_{0}\right) \mathrm{e}^{-l \Omega_{0} t}, \\
\Omega_{0}=\omega\left(k_{0}\right)+\frac{1}{2} T_{\mathbf{k}_{0}}|A|^{2}, \\
T_{\mathbf{k}_{0}}=T_{\mathbf{k}_{0} \mathbf{k}_{0} \mathbf{k}_{0} \mathbf{k}_{0}}=\frac{1}{2 \pi} k_{0}^{3} .
\end{gathered}
$$

This solution is nothing but the stationary Stokes wave. It gives the right expression for the two first terms in series (1) and (2).

Equation (41) has also a reach set of approximate quasi-periodic solutions. Let $\kappa_{1}$ and $\kappa_{2}$ are two arbitrary wave vectors. In the limit of small $\left|A_{1}\right|^{2},\left|A_{2}\right|^{2}$ equation has the following solution:

$$
\begin{aligned}
& b_{\mathbf{k}}=A_{1} \delta\left(\mathbf{k}-\kappa_{1}\right) \mathrm{e}^{-l \tilde{\Omega}_{1} t}+A_{2} \delta\left(\mathbf{k}-\kappa_{2}\right) \mathrm{e}^{-l \tilde{\Omega}_{2} t}+\ldots \\
& \tilde{\Omega}_{1}=\omega\left(\kappa_{1}\right)+\frac{1}{2} T_{\kappa_{1}}\left|A_{1}\right|^{2}+T_{\kappa_{1}, \kappa_{2}}\left|A_{2}\right|^{2}, \\
& \tilde{\Omega}_{2}=\omega\left(\kappa_{2}\right)+T_{\kappa_{1}, \kappa_{2}}\left|A_{1}\right|^{2}+\frac{1}{2} T_{\kappa_{1}}\left|A_{2}\right|^{2} .
\end{aligned}
$$

Here $T_{\kappa_{1}, \kappa_{2}}=T_{\kappa_{1} \kappa_{2}, \kappa_{1} \kappa_{2}}$. Equations (43), (44) are valid if nonlinear terms in (44) are much less that linear. 
In the particular case $\kappa_{2}=-\kappa_{1},\left|A_{2}\right|=\left|A_{1}\right|$, the solution (43) is just a standing wave.

Both propagating wave (42) and standing wave (43) are unstable. To study the instability of propagating wave (42), we will look for a solution in the following form

$$
b_{\mathbf{k}}=A_{0} \delta\left(\mathbf{k}-\mathbf{k}_{0}\right) \mathrm{e}^{-l \Omega_{0} t}+\alpha \delta\left(\mathbf{k}-\mathbf{k}_{0}-\kappa\right) \mathrm{e}^{-l \Omega_{1} t}+\beta \delta\left(\mathbf{k}-\mathbf{k}_{0}+\kappa\right) \mathrm{e}^{-l \Omega_{2} t} .
$$

Here

$$
\begin{aligned}
& \Omega_{1}=\omega\left(\mathbf{k}_{0}+\kappa\right)+2 T\left(\mathbf{k}_{0}, \mathbf{k}_{0}+\kappa\right)|A|^{2}, \\
& \Omega_{2}=\omega\left(\mathbf{k}_{0}-\kappa\right)+2 T\left(\mathbf{k}_{0}, \mathbf{k}_{0}-\kappa\right)|A|^{2} .
\end{aligned}
$$

By plugging (43) into (41) and linearizing over $\alpha$ and $\beta$ we set system of ordinary differential equations, similar to (33)

$$
\begin{aligned}
& \dot{\alpha}=\frac{l}{2} \mathrm{e}^{-l \Delta}\left|A_{0}\right|^{2} T \beta^{*}, \quad T=T_{\mathbf{k}_{0}, \mathbf{k}_{0}, \mathbf{k}_{0}+\kappa, \mathbf{k}_{0}-\kappa}, \\
& \dot{\beta}=\frac{l}{2} \mathrm{e}^{-l \Delta}\left|A_{0}\right|^{2} T \alpha^{*}, \\
& \Delta=2 \omega\left(k_{0}\right)-\omega\left(\mathbf{k}_{0}-\kappa\right)-\omega\left(\mathbf{k}_{0}+\kappa\right)+2\left(T_{\mathbf{k}_{0}}-T_{\mathbf{k}_{0}, \kappa_{1}}-T_{\mathbf{k}_{0}, \kappa_{2}}\right)\left|A_{0}\right|^{2} .
\end{aligned}
$$

Solutions of equation (47) are given by formulae (34) where

$$
\gamma=\sqrt{|A|^{4}|T|^{2}-\frac{1}{4} \Delta^{2}}
$$

One can see that instability occurs in a vicinity of the curve $\Delta=0$. For waves on a deep water, we can put $\mathbf{k}_{0}=\mathbf{i}, \kappa=x \mathbf{i}+y \mathbf{j}$. Then the condition $\Delta=0$ is reduced to the famous Phillips curve [29]

$$
\left[(1+x)^{2}+y^{2}\right]^{1 / 4}+\left[(1-x)^{2}+y^{2}\right]^{1 / 4}=2 .
$$

Here $-5 / 4 \leq x \leq 5 / 4$. The Phillips curve is plotted in Figure 3. The coupling coefficient $T\left(\mathbf{k}_{0}, x\right)$, evaluated on the Phillips curve, can be presented in the form

$$
T\left(2 \mathbf{k}_{0}, \mathbf{k}_{0}+\kappa, \mathbf{k}_{0}-\kappa\right)=k_{0}^{3} f(x), \quad x=\frac{\kappa_{x}}{k_{0}},
$$

$f(-x)=f(x)$ symmetric function. It is important to mention that $f(5 / 4)=0$. This fact was first discovered by Dyachenko and Zakharov in 1994 [30]. The decrease of $f(x)$ with growth of $x$ means that the four-wave instability is mostly modulational because the most unstable modes are concentrated at $\kappa \rightarrow 0$. In this region

$$
\gamma \simeq \frac{1}{2} \sqrt{-2 T|A|^{2} \frac{\partial^{2} \omega}{\partial k_{\alpha} \partial k_{\beta}} \kappa_{\alpha} \kappa_{\beta}-\left(\frac{\partial^{2} \omega}{\partial k_{\alpha} \partial k_{\beta}} \kappa_{\alpha} \kappa_{\beta}\right)^{2}} .
$$




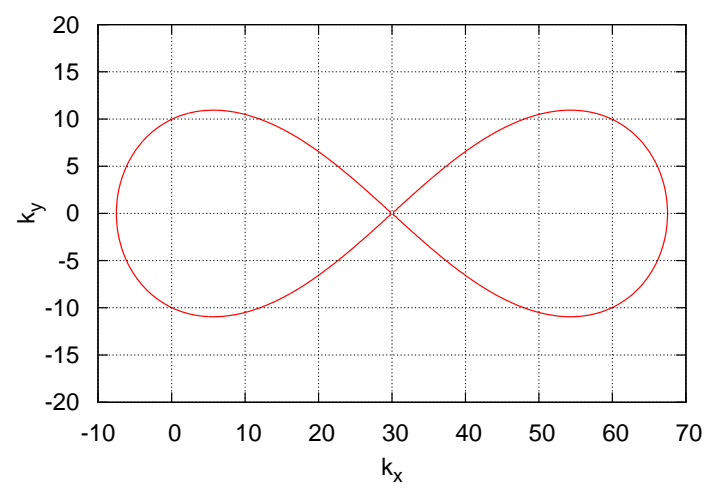

Figure 3: Phillips curve for $\mathbf{k}_{0}=(30,0)$.

Instability is concentrated inside the angle where

$$
\frac{\partial^{2} \omega}{\partial k_{\alpha} \partial k_{\beta}} \kappa_{\alpha} \kappa_{\beta}<0 .
$$

The plot of growth rate (48) on a homogeneous grid of wave numbers for $2 \pi \times$ $2 \pi$ periodic box is given in Figure 4. The four-wave instability of propagating
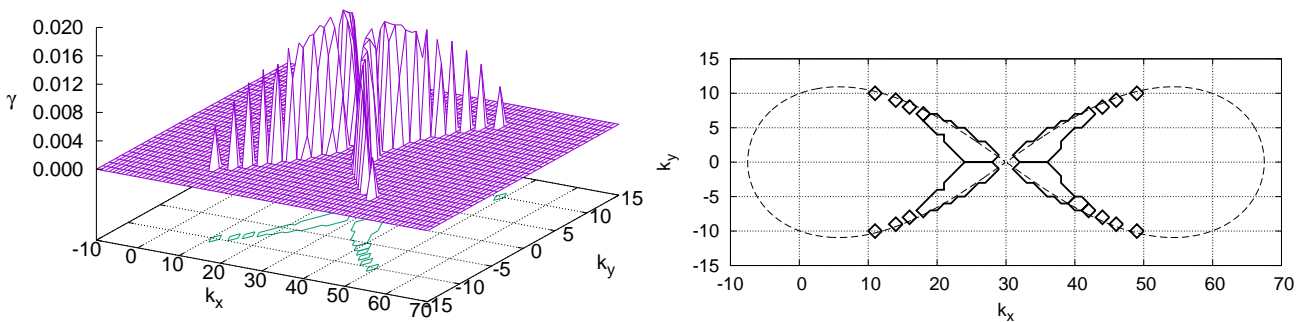

Figure 4: Growth rate for Phillips instability of monochromatic gravity wave with $\mathbf{k}_{0}=(30,0)$ and average steepness $\mu=0.1$ on the homogeneous grid. Left panel: isometric projection; right panel: contour of the surface at the level $10^{-23}$; dashed line - resonance curve.

Stokes waves was studied in detail in many papers (e. g. $[9,10,11,12,13,14$, 31]).

Let us study instability of by-harmonic wave (45). We concentrate only on the case of standing wave $\kappa_{2}=-\kappa_{1}=k_{0}$. The standing wave is unstable due to a different mechanism. Firstly, each propagating wave composing the standing wave endure its own modulational instability. Then, another instability appears. In this new type of instability, we have the simultaneous excitation of two waves with wave numbers $\pm \kappa$ such that $|\kappa|=\left|k_{0}\right|$. The maximal growth-rate of this 
instability is:

$$
\gamma_{\max } \simeq \frac{1}{2} \tilde{T}|A|^{2} .
$$

Here

$$
\tilde{T}=T_{\mathbf{k}_{0},-\mathbf{k}_{0}, \kappa,-\kappa}=k_{0}^{3} f(\cos \theta),
$$

and $\theta$ is an angle between $\mathbf{k}_{0}$ and $\kappa$.

\section{Numerical Simulation Scheme.}

The problem of numerical integration of system of equations (18) is rather complicated. One of the most important questions is what time integration algorithm to choose. According to the property of the equations, it would be natural to develop a numerical integration scheme conserving the Hamiltonian. Let us follow the article [32]. One can introduce a discrete variation of Hamiltonian (16) in one step on time $H^{n} \longrightarrow H^{n+1}$

$$
\Delta H=H^{n+1}-H^{n} .
$$

The Hamiltonian is a function of canonical variables $\eta$ and $\psi$. The discrete variations of these functions on a time step are equal to

$$
\Delta \eta=\eta^{n+1}-\eta^{n}, \quad \Delta \psi=\psi^{n+1}-\psi^{n} .
$$

One can expand discrete variation $\Delta H$ via $\Delta \eta$ and $\Delta \psi$ (that is done in Appendix A)

$$
\Delta H=H_{\psi} \Delta \psi+H_{\eta} \Delta \eta .
$$

It is easy to see that $H_{\eta}$ and $H_{\psi}$ are discrete analogues to the continuous variations $\frac{\delta H}{\delta \eta}$ and $\frac{\delta H}{\delta \psi}$.

One can demand the conservation of Hamiltonian $\Delta H / \tau=0$ during time step $\tau$. Obviously, this equality can take place if the following conditions are valid:

$$
\begin{gathered}
\frac{\Delta \eta}{\tau}=H_{\psi}, \\
\frac{\Delta \psi}{\tau}=-H_{\eta} .
\end{gathered}
$$

In some sense, this is a discrete analogue of Hamiltonian equations (15). Thus, if Hamiltonian variation (52) is expanded via variations $\Delta \eta$ and $\Delta \psi$, it is possible to get equations (55). 
As it was mentioned above, it is more convenient to rewrite the equations in terms of Fourier harmonics. Using the results given in Appendix A A.2 A.7), one can obtain an implicit difference scheme

$$
\begin{aligned}
& \frac{\eta_{\mathbf{k}}^{n+1}-\eta_{\mathbf{k}}^{n}}{\tau}=\frac{1}{2}|\mathbf{k}|\left(\psi_{\mathbf{k}}^{n+1}+\psi_{\mathbf{k}}^{n}\right)- \\
& -\frac{1}{4} \hat{F}\left(\nabla,\left(\eta^{n+1}+\eta^{n}\right) \nabla\left(\psi^{n+1}+\psi^{n}\right)\right)- \\
& -\frac{1}{4}|\mathbf{k}| \hat{F}\left(\left(\eta^{n+1}+\eta^{n}\right) \hat{k}\left(\psi^{n+1}+\psi^{n}\right)\right)+ \\
& +\frac{1}{4}|\mathbf{k}| \hat{F}\left[\left(\eta^{n+1}+\eta^{n}\right) \hat{k}\left(\eta^{n+1} \hat{k} \psi^{n+1}+\eta^{n} \hat{k} \psi^{n}\right)\right]- \\
& -\frac{1}{8}|\mathbf{k}|^{2} \hat{F}\left[\left(\left(\eta^{n+1}\right)^{2}+\left(\eta^{n}\right)^{2}\right) \hat{k}\left(\psi^{n+1}+\psi^{n}\right)\right]+ \\
& +\frac{1}{8}|\mathbf{k}| \hat{F}\left[\left(\left(\eta^{n+1}\right)^{2}+\left(\eta^{n}\right)^{2}\right) \nabla^{2}\left(\psi^{n+1}+\psi^{n}\right)\right] \text {. } \\
& \frac{\psi_{\mathbf{k}}^{n+1}-\psi_{\mathbf{k}}^{n}}{\tau}=-\frac{1}{2} \frac{\omega_{k}^{2}}{|\mathbf{k}|}\left(\eta_{\mathbf{k}}^{n+1}+\eta_{\mathbf{k}}^{n}\right)- \\
& -\frac{1}{4} \hat{F}\left(\left|\nabla \psi^{n+1}\right|^{2}+\left|\nabla \psi^{n}\right|^{2}\right)+ \\
& +\frac{1}{4} \hat{F}\left(\left(\hat{k} \psi^{n+1}\right)^{2}+\left(\hat{k} \psi^{n}\right)^{2}\right)- \\
& -\frac{1}{4} \hat{F}\left[\hat{k}\left(\psi^{n+1}+\psi^{n}\right) \hat{k}\left(\eta^{n+1} \hat{k} \psi^{n+1}+\eta^{n} \hat{k} \psi^{n}\right)\right]- \\
& -\frac{1}{4} \hat{F}\left[\left(\eta^{n+1}+\eta^{n}\right)\left(\nabla^{2} \psi^{n+1} \hat{k} \psi^{n+1}+\nabla^{2} \psi^{n} \hat{k} \psi^{n}\right)\right] .
\end{aligned}
$$

Here, $\hat{F}$ is the Fourier transform operator.

It is useful to resolve the linear part of scheme (56-57) with respect to $\eta^{n+1}$ and $\psi^{n+1}$. Let us denote nonlinear terms on the right hand sides of these equations as:

$$
\begin{aligned}
R_{\eta}^{n+1}= & -\frac{1}{4} \hat{F}\left(\nabla,\left(\eta^{n+1}+\eta^{n}\right) \nabla\left(\psi^{n+1}+\psi^{n}\right)\right)- \\
& -\frac{1}{4}|\mathbf{k}| \hat{F}\left(\left(\eta^{n+1}+\eta^{n}\right) \hat{k}\left(\psi^{n+1}+\psi^{n}\right)\right)+ \\
& +\frac{1}{4}|\mathbf{k}| \hat{F}\left[\left(\eta^{n+1}+\eta^{n}\right) \hat{k}\left(\eta^{n+1} \hat{k} \psi^{n+1}+\eta^{n} \hat{k} \psi^{n}\right)\right]- \\
& -\frac{1}{8}|\mathbf{k}|^{2} \hat{F}\left[\left(\left(\eta^{n+1}\right)^{2}+\left(\eta^{n}\right)^{2}\right) \hat{k}\left(\psi^{n+1}+\psi^{n}\right)\right]+ \\
& +\frac{1}{8}|\mathbf{k}| \hat{F}\left[\left(\left(\eta^{n+1}\right)^{2}+\left(\eta^{n}\right)^{2}\right) \nabla^{2}\left(\psi^{n+1}+\psi^{n}\right)\right], \\
R_{\psi}^{n+1}= & -\frac{1}{4} \hat{F}\left(\left|\nabla \psi^{n+1}\right|^{2}+\left|\nabla \psi^{n}\right|^{2}\right)+ \\
& +\frac{1}{4} \hat{F}\left(\left(\hat{k} \psi^{n+1}\right)^{2}+\left(\hat{k} \psi^{n}\right)^{2}\right)- \\
& -\frac{1}{4} \hat{F}\left[\hat{k}\left(\psi^{n+1}+\psi^{n}\right) \hat{k}\left(\eta^{n+1} \hat{k} \psi^{n+1}+\eta^{n} \hat{k} \psi^{n}\right)\right]- \\
& -\frac{1}{4} \hat{F}\left[\left(\eta^{n+1}+\eta^{n}\right)\left(\nabla^{2} \psi^{n+1} \hat{k} \psi^{n+1}+\nabla^{2} \psi^{n} \hat{k} \psi^{n}\right)\right] .
\end{aligned}
$$

Using these notations, discrete scheme can be written as follows

$$
\begin{aligned}
& \eta_{\mathbf{k}}^{n+1}=A(k, \tau) \eta_{\mathbf{k}}^{n}+B(k, \tau) \psi_{\mathbf{k}}^{n}+C(k, \tau) R_{\eta}^{n+1}+D(k, \tau) R_{\psi}^{n+1} \\
& \psi_{\mathbf{k}}^{n+1}=E(k, \tau) \eta_{\mathbf{k}}^{n}+A(k, \tau) \psi_{\mathbf{k}}^{n}+F(k, \tau) R_{\eta}^{n+1}+C(k, \tau) R_{\psi}^{n+1}
\end{aligned}
$$


Here

$$
\begin{aligned}
& A(k, \tau)=\frac{1-\frac{1}{4} \omega_{k}^{2} \tau^{2}}{1+\frac{1}{4} \omega_{k}^{2} \tau^{2}}, \quad B(k, \tau)=\frac{\tau k}{1+\frac{1}{4} \omega_{k}^{2} \tau^{2}}, \\
& C(k, \tau)=\frac{\tau}{1+\frac{1}{4} \omega_{k}^{2} \tau^{2}}, \quad D(k, \tau)=\frac{1}{2} \tau B(k, \tau), \\
& E(k, \tau)=-\frac{\omega_{k}^{2}}{k} C(k, \tau), \quad F(k, \tau)=\frac{1}{2} \tau E(k, \tau) .
\end{aligned}
$$

Thus, we get implicit (terms $R_{\eta}^{n+1}$ and $R_{\psi}^{n+1}$ contain $\eta_{\mathbf{k}}^{n+1}$ and $\psi_{\mathbf{k}}^{n+1}$ ) difference scheme. The important feature of this scheme is that conservation of Hamiltonian (12)-(13) is embedded in it.

The implicit numerical scheme (59) can be solved by the method of FixedPoint Iterations [33]. Let us write this procedure for $\eta_{\mathbf{k}}^{n+1, s}$ and $\psi_{\mathbf{k}}^{n+1, s}$, here $s$ is an iteration number. Corresponding to (59) one can get

$$
\begin{aligned}
& \eta_{\mathbf{k}}^{n+1,0}=\eta_{\mathbf{k}}^{n}, \psi_{\mathbf{k}}^{n+1,0}=\psi_{\mathbf{k}}^{n} ; \\
& \eta_{\mathbf{k}}^{n+1, s+1}=A(k, \tau) \eta_{\mathbf{k}}^{n}+B(k, \tau) \psi_{\mathbf{k}}^{n}+C(k, \tau) R_{\eta}^{n+1, s}+D(k, \tau) R_{\psi}^{n+1, s}, \\
& \psi_{\mathbf{k}}^{n+1, s+1}=E(k, \tau) \eta_{\mathbf{k}}^{n}+A(k, \tau) \psi_{\mathbf{k}}^{n}+F(k, \tau) R_{\eta}^{n+1, s}+C(k, \tau) R_{\psi}^{n+1, s} .
\end{aligned}
$$

Iterations continue until the desired accuracy of Hamiltonian conservation $\epsilon$ is achieved. Convergence is ensured by the choice of time step $\tau$ to be small enough. The sufficient condition for the convergence of iterations is the following:

$$
\tau^{2}<\frac{1}{\max \left[(\nabla \psi)^{2}+(\hat{k} \psi)^{2}\right] k^{2}-1 / 4 \min \left(\omega_{k}^{2}\right)} .
$$

Taking into account that the first factor in the first term in the denominator of (62) is of the order of maximum velocity on the surface, this condition is similar to $\tau<\Delta x / v_{\max }$. We resolved the linear part of the equation which is why frequency doesn't come into play.

In most cases, it is enough to follow the convergence of the relative error

$$
\frac{\sum_{\mathbf{k}}\left|\eta_{\mathbf{k}}^{n+1, s+1}\right|^{2}-\sum_{\mathbf{k}}\left|\eta_{\mathbf{k}}^{n+1, s}\right|^{2}}{\sum_{\mathbf{k}}\left|\eta_{\mathbf{k}}^{n+1, s}\right|^{2}}<\epsilon .
$$

When studying gravity waves, this condition is equivalent to the calculation of potential energy with desired accuracy. For the weakly nonlinear regime, the 
quadratic part of Hamiltonian is dominant, so the physical meaning of this condition is quite clear.

The condition on time step (62) is not very practical to use. It is much easier to control step on time, demanding convergence of the iteration process to the desired accuracy after a number of iterations less than some given $N_{\max }$ and more than some given $N_{\min }$.

This numerical scheme can be used for the simulation of freely decaying waves. For the simulation of turbulence, we should introduce pumping and damping terms in the equations. It is possible to do that through different ways, but we have applied the Split-Step Method, which is very popular in the numerical simulation of pulse propagation in optic fibers. Here is a very brief description of application of this method to our problem.

Let us introduce a linear damping with rate $\gamma_{k}$ into our model

$$
\dot{\psi}_{\mathbf{k}}=\text { R.H.S. }-\gamma_{k} \psi_{\mathbf{k}} .
$$

It is possible to take into account this damping without significant changes in the calculations scheme. First, one can obtain a solution of equations (18) without damping using iteration scheme described above. Let us denote this solution by $\tilde{\psi}_{\mathbf{k}}^{n+1}$. Second, the solution of the whole system of equations can be calculated by the next step

$$
\psi_{\mathbf{k}}^{n+1}=\tilde{\psi}_{\mathbf{k}}^{n+1} \exp \left(-\gamma_{k} \tau\right)
$$

It is worthwhile to say that for weak turbulence simulation the most interesting part of the spectrum is in the "inertial interval" where there is no damping or pumping at all. Even more, the nature of damping and pumping is not important. In this case, influence of non-conservative terms can be described by such a rough scheme. As a bonus, we have eliminated the restrictions on time step

$$
\max \left(\left|\gamma_{k}\right|\right) \tau<1
$$

which would be unavoidable in the case of integration by standard Runge-Kutta methods. Pumping can be considered in a similar way.

\section{Capillary waves}

In this section, we briefly review our previous results published in [18] and report new observations. The system of equations (18) was simulated in the domain $L_{x}=L_{y}=2 \pi$, with surface tension coefficient $\sigma=1$. The number 
of grid points was $512 \times 512$. A monochromatic wave of amplitude $\left|a_{\mathbf{k}_{0}}\right|=$ $2 \times 10^{-3}$, which corresponds to average steepness $\mu=0.05$, was taken as the initial condition. Its wave number vector $\mathbf{k}_{0}=(68,0)$. All other harmonics were of amplitude $\left|a_{\mathbf{k}}\right| \sim 10^{-12}$ and with random phase (Figure 5). As was mentioned

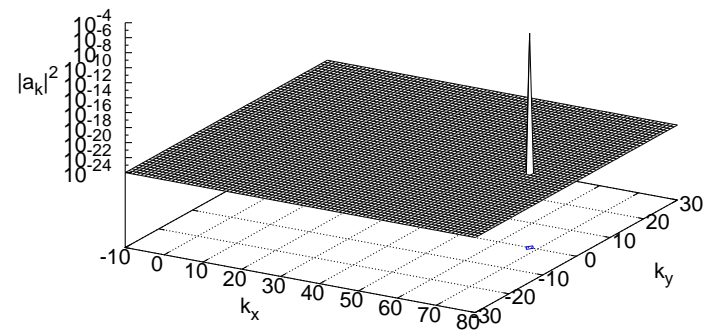

Figure 5: Decay of the monochromatic capillary wave. Initial conditions. Time $t=0$.

above, resonant curve almost never passes through grid points (there are two non trivial points $\mathbf{k}=(0 ; 0)$ and initial wave $\mathbf{k}=\left(k_{0}, 0\right)$; this process has a zero growth rate). A detailed picture of the resonant curve on the grid in the region with the highest grid point density in the vicinity of the curve is shown in Figure 6. One can see that some points are closer to the resonant curve than others.

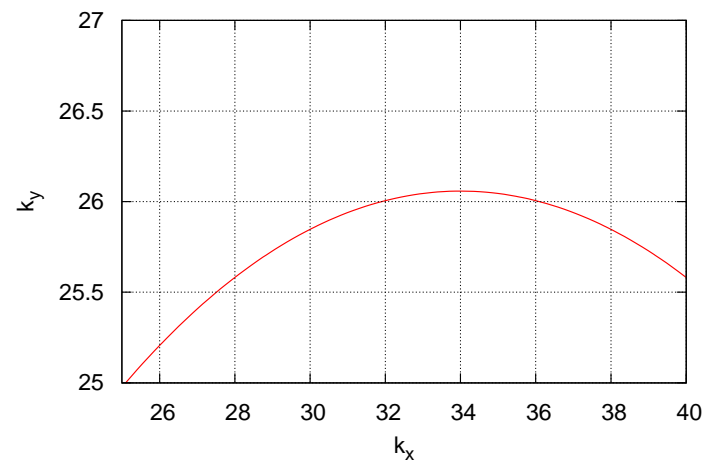

Figure 6: Part of the resonant curve for the decay of the monochromatic capillary wave with $\mathbf{k}_{0}=(68,0)$. The different mismatches for different grid knots are clearly seen.

In the beginning one can see the growth of several harmonics as it is predicted in (34) and (35). Different stages of the decay process are represented in Figures 7, 10 and time is given in periods of initial wave $T_{0}$. We represent the isometric projection of the $\left|a_{\mathbf{k}}\right|^{2}$-surface and contour of this surface at the level $10^{-23}$ (order of magnitude higher than background noise). The full picture of the $k$-plane in the final moment of simulations is represented in Figure 11, A 

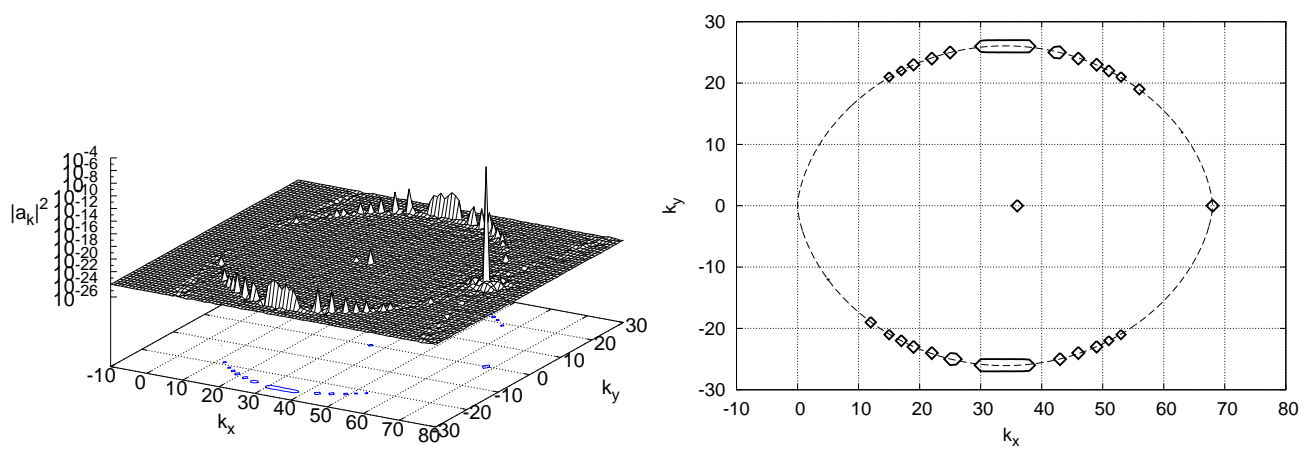

Figure 7: Decay of the monochromatic capillary wave. Growth of the harmonics in the vicinity of the resonant curve has begun. Time $t=318 T_{0}$.
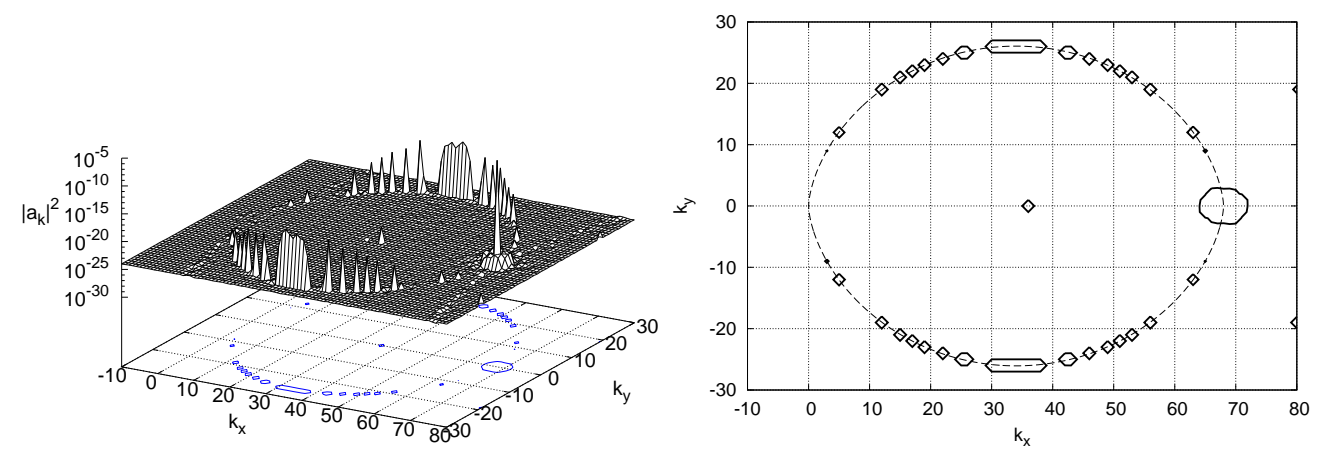

Figure 8: Decay of the monochromatic capillary wave. Decay harmonics are well developed. Time $t=794 T_{0}$.
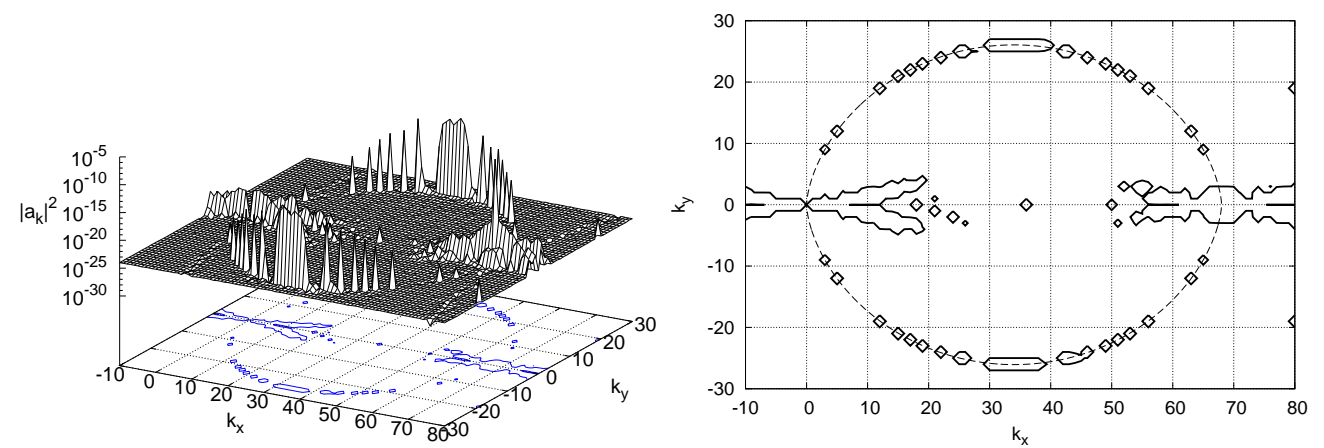

Figure 9: Decay of the monochromatic capillary wave. Secondary nonlinear processes are revealed. Time $t=1112 T_{0}$. 

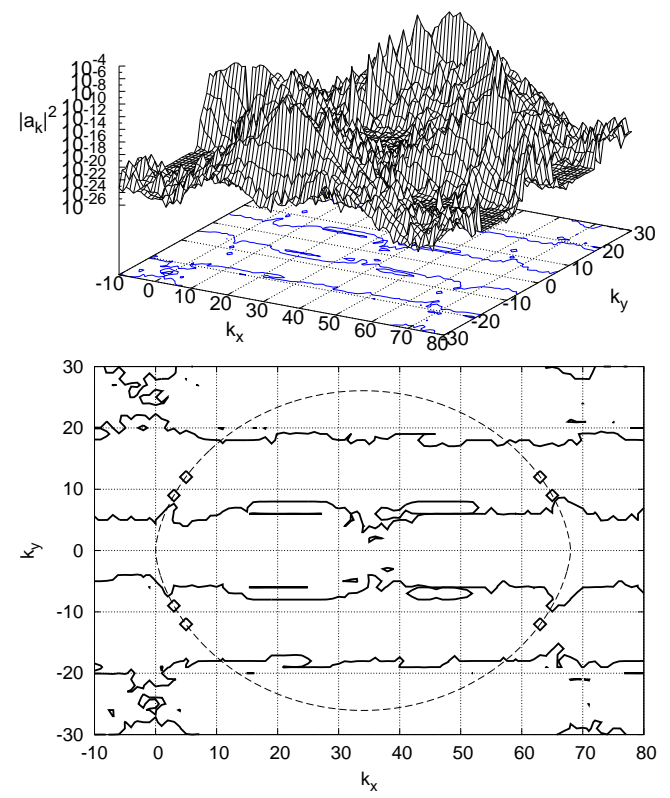

Figure 10: Decay of the monochromatic capillary wave. Secondary nonlinear processes are well developed. Time $t=1589 T_{0}$.

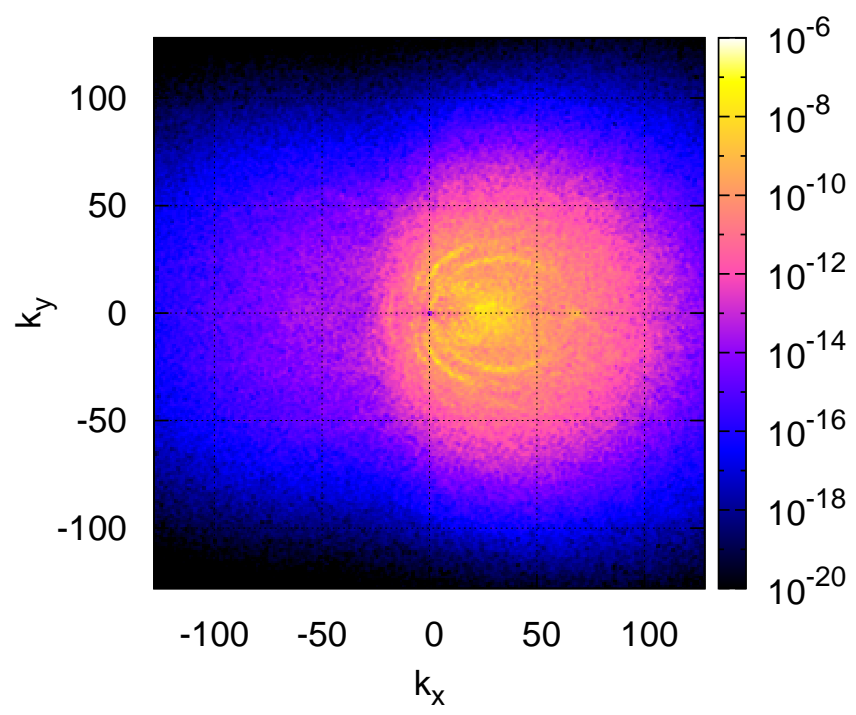

Figure 11: Decay of the monochromatic capillary wave. Full $k$-plane. Time $t=144488 T_{0}$. 
closer snapshot of the most interesting region of Figure 11(initial decay region) is represented in Figure 12. One can see that, although the amplitudes of the

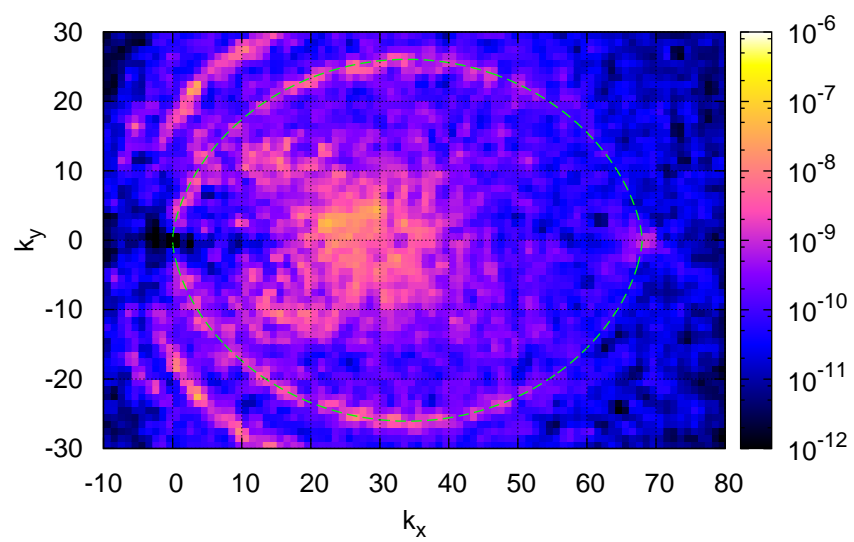

Figure 12: Decay of the monochromatic capillary wave. A closer snapshot of the initial decay region. Time $t=144488 T_{0}$.

waves are stochastic, the spectrum is still strongly anisotropic. 


\section{Gravity waves.}

In the case of gravity waves on the surface of deep fluid, the dispersion is the following

$$
\omega_{k}=\sqrt{g k},
$$

here $g$ is a gravity acceleration. Here and further, let us suppose $g=1$.

In this case, dispersion is of non-decay type conditions (37) have no real nontrivial solutions, and the main process is four-wave scattering. Therefore, one can make a substitution to eliminate third order terms corresponding to the decay process. This is the reason why we have to use Hamiltonian expansion up to forth order in the case of gravity waves.

Let us consider the same initial conditions as in the case of the decay of the monochromatic capillary wave (i.e. one monochromatic wave and random phase noise of small amplitude). The main processes correspond to the cases when a large amplitude of initial wave involved the highest possible number of times. In this case, one wave to three and inverse processes are much weaker than scattering of two waves with the same amplitude and the same wave vector to two other waves.

Resonance conditions for such process are as follows:

$$
\omega_{k_{1}}+\omega_{k_{2}}=2 \omega_{k_{0}}, \quad \mathbf{k}_{1}+\mathbf{k}_{2}=2 \mathbf{k}_{0} .
$$

The resonant curve for this conditions is shown in Figure 3 ,

The system of equations (18) was simulated in domain $L_{x}=L_{y}=2 \pi$ with gravity acceleration $g=1$. Grid size was equal to $512 \times 512$ points. As an initial condition, monochromatic wave of amplitude $\left|a_{\mathbf{k}_{0}}\right|=1.3 \times 10^{-3}$ with wave number vector $\mathbf{k}_{0}=(30,0)$ was used. All other harmonics were of amplitude $\left|a_{\mathbf{k}}\right| \sim 10^{-12}$ and with random phase (Figure 13). In the beginning, one can

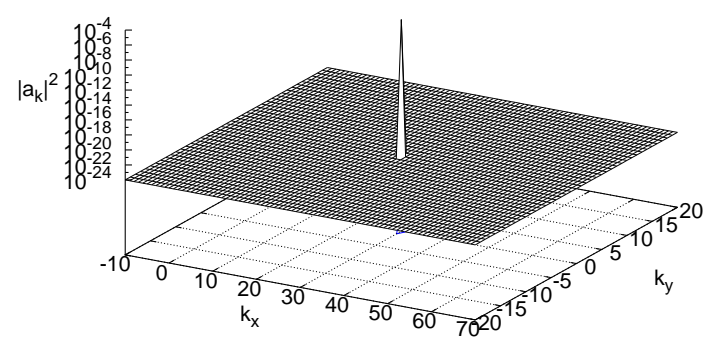

Figure 13: Instability of the monochromatic gravity wave. Initial condition. Time $t=0$. 
observe exponential growth of several harmonics in the vicinity of the resonant curve (a detailed picture of resonant curve in the surroundings of the initial wave is shown in Figure 14). This is shown in Figure 15. It is clearly seen that the wave with wavevector $(33,2)$ has smallest mismatch and, as a result, growth occurs. As we already know, four-wave scattering growth-rate has the highest values in

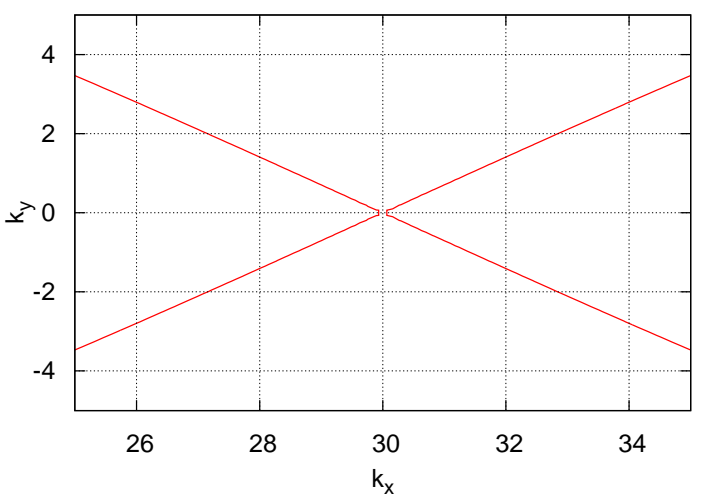

Figure 14: Gravity waves. Part of the resonant curve. Different mismatch for different grid points is clearly visible.

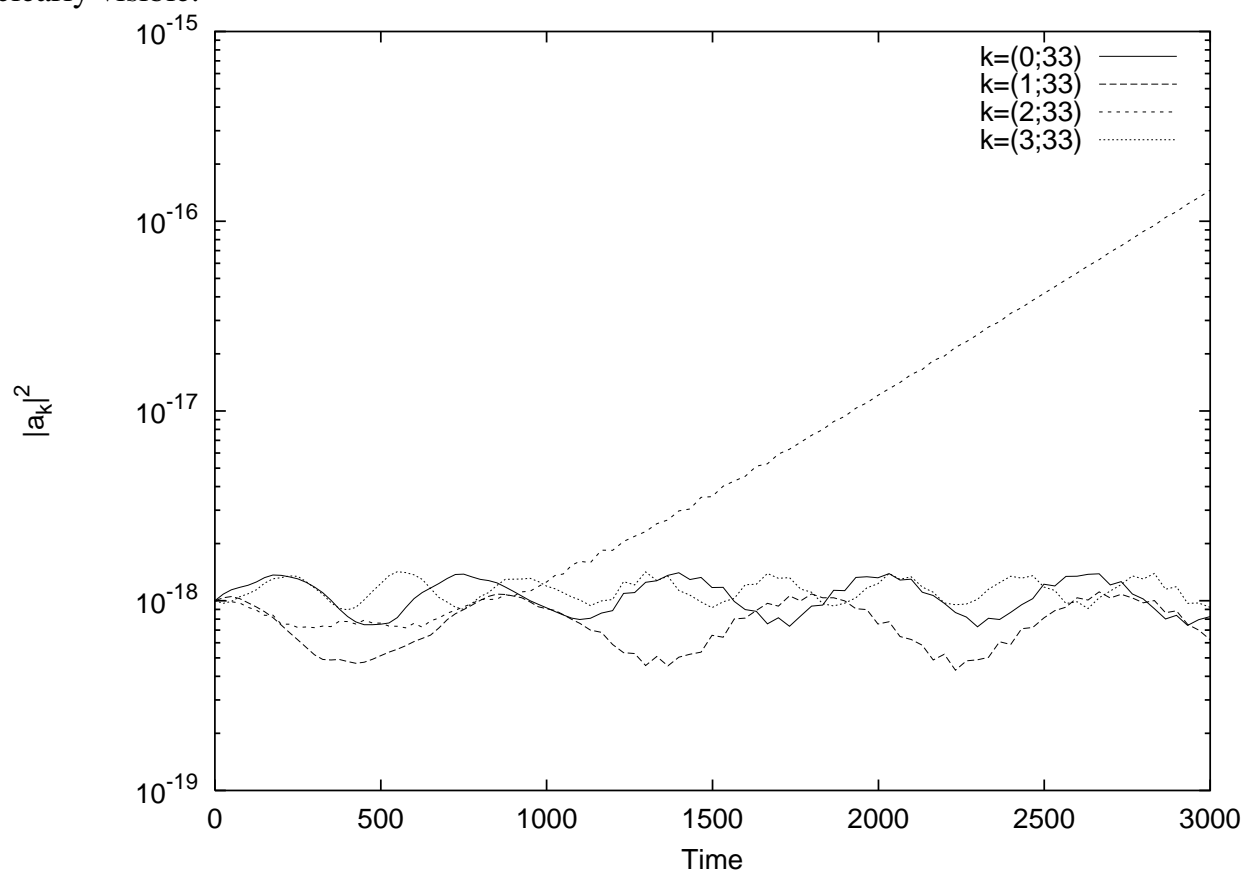

Figure 15: Gravity waves. Growth of harmonics amplitude as a function of time. One can see that harmonic $\mathbf{k}=(33,2)$ is in almost exact resonance, and the others are not. 
the vicinity of $\mathbf{k}_{0}=\left(k_{0}, 0\right)$. Due to this, the initial growth is concentrated about the cross of the resonant manifold. Different stages are represented in Figures 16 21. We represent the isometric projection of the $\left|a_{\mathbf{k}}\right|^{2}$-surface and contour of this surface at the level $10^{-23}$ (order of magnitude higher than background noise).
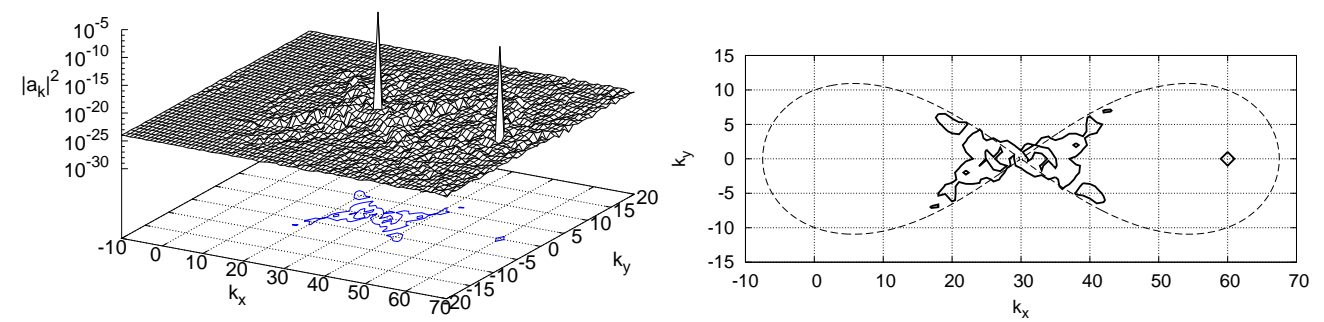

Figure 16: Instability of the monochromatic gravity wave. Growth of the harmonics in the vicinity of the resonant curve has began. Time $t=43 T_{0}$.
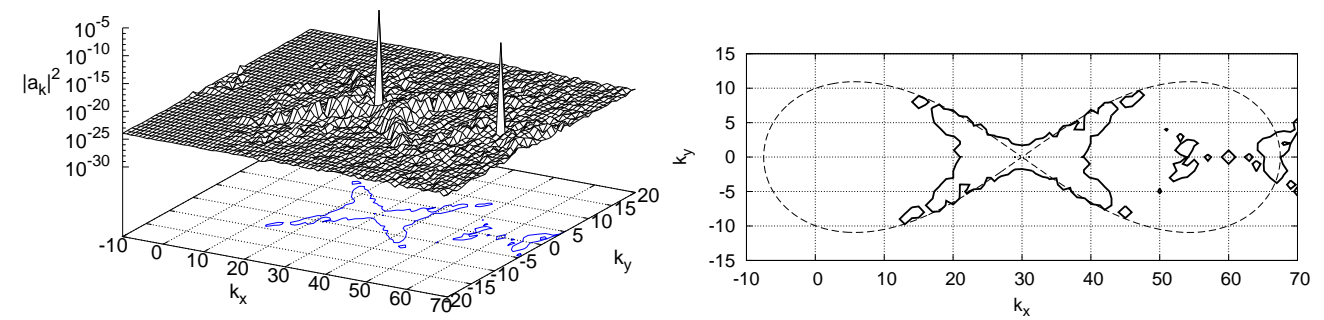

Figure 17: Instability of the monochromatic gravity wave. Growth of the harmonics in the vicinity of the resonant curve continues. Time $t=87 T_{0}$.
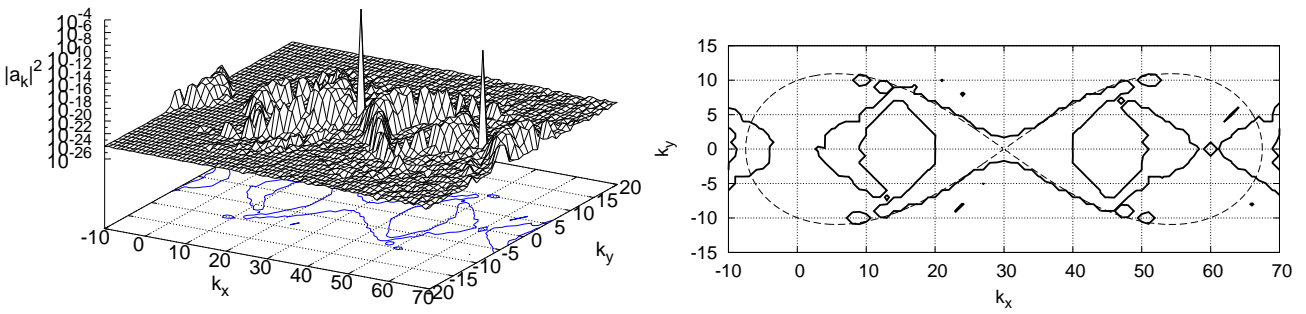

Figure 18: Instability of the monochromatic gravity wave. Harmonics on the resonant curve are well developed. Time $t=174 T_{0}$.

The full picture of the $k$-plane in the final moment of simulations is represented in Figure 22. A closer snapshot of the most interesting region of Figure22(initial instability region) is represented in Figure 23. One can observe a still weak but visible downshift of the spectrum. 

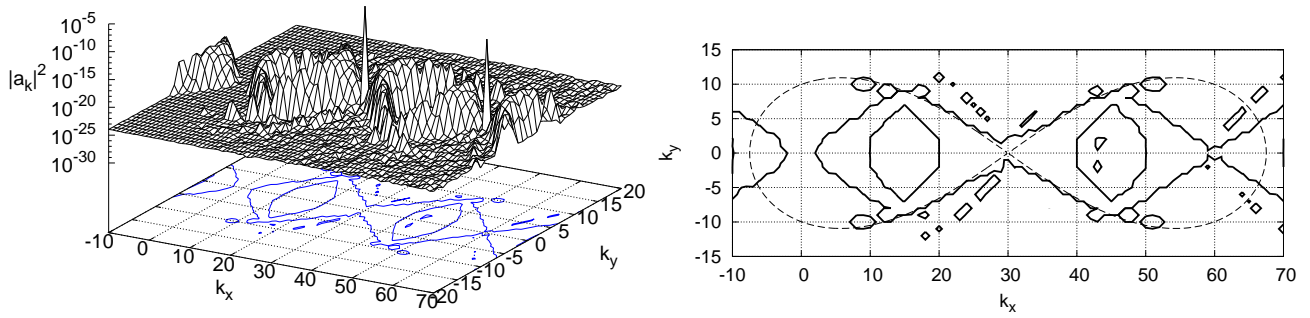

Figure 19: Instability of the monochromatic gravity wave. Beginning of the secondary processes. Time $t=261 T_{0}$.
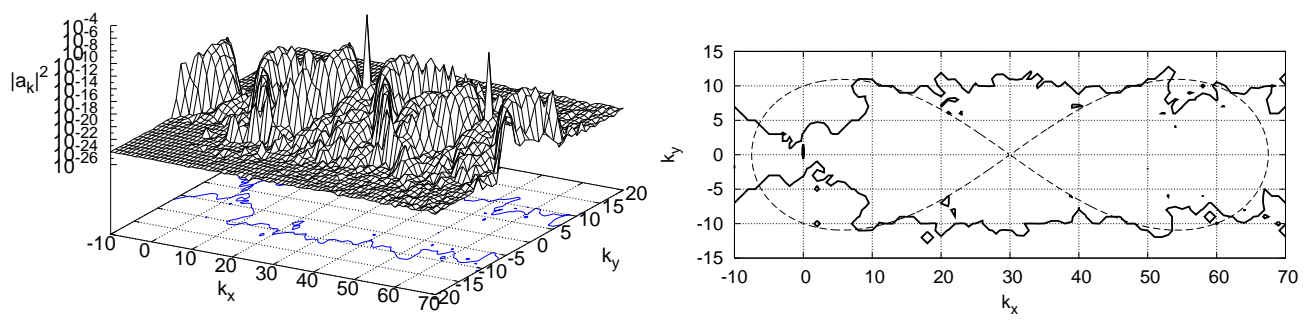

Figure 20: Instability of the monochromatic gravity wave. Secondary processes are well developed. Time $t=348 T_{0}$.
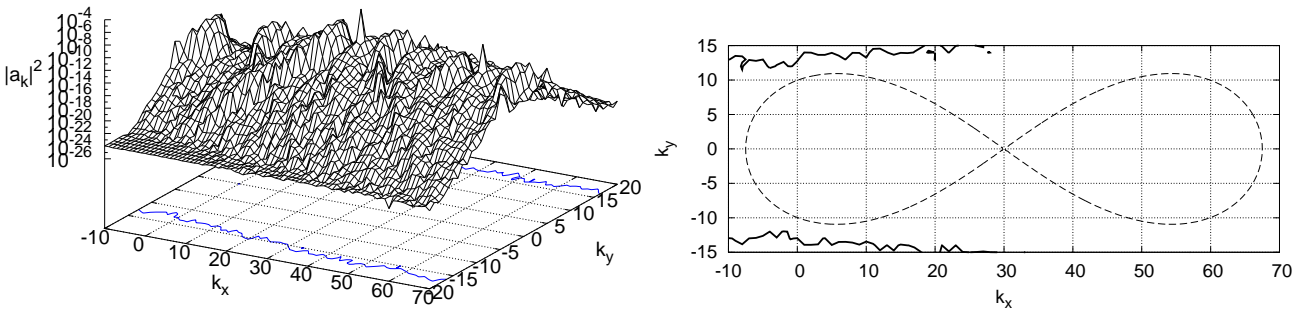

Figure 21: Instability of the monochromatic gravity wave. Secondary processes hide the structure of the resonances. Time $t=435 T_{0}$. 


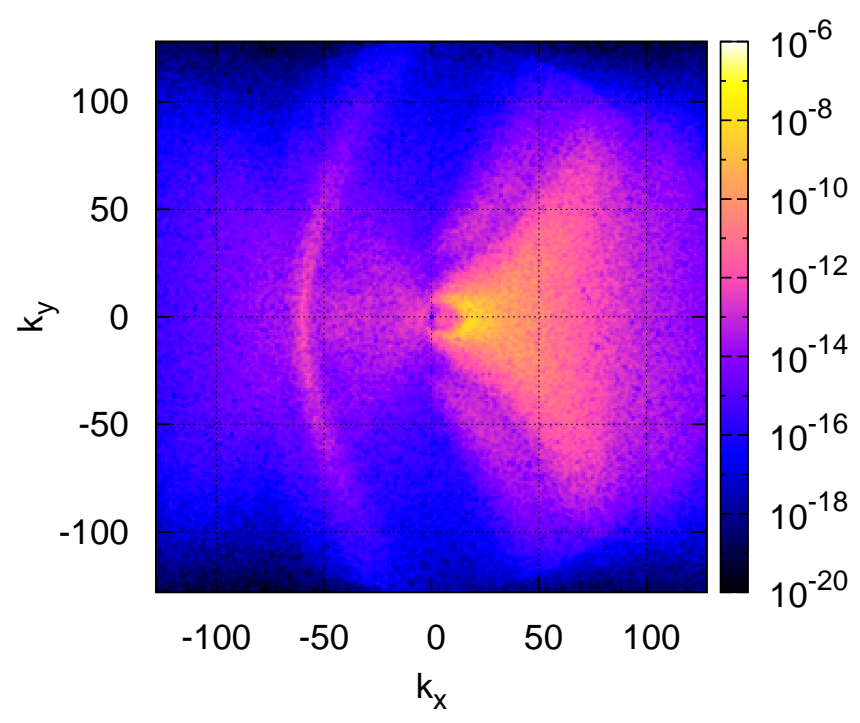

Figure 22: Instability of the monochromatic gravity wave. Full $k$-plane. Time $t=1204 T_{0}$.

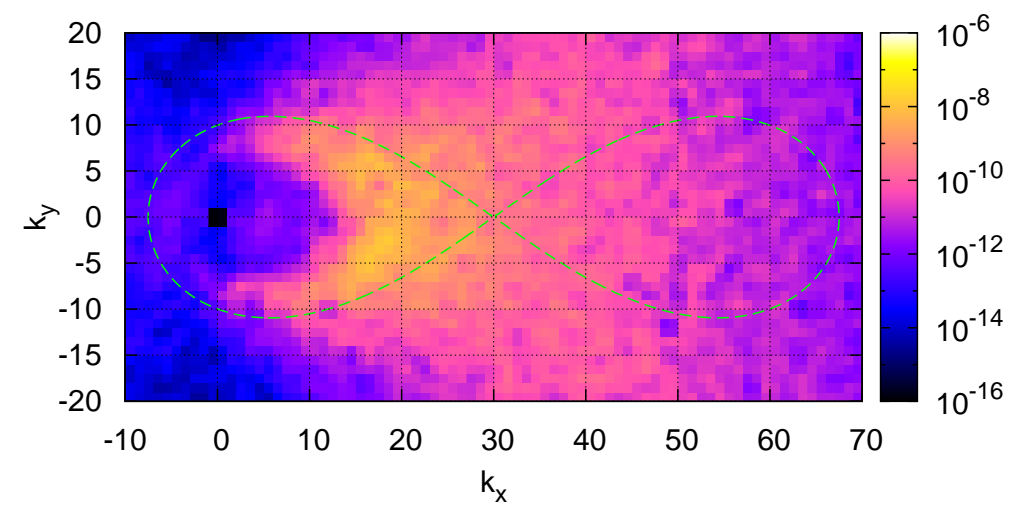

Figure 23: Decay of the monochromatic capillary wave. A closer snapshot of the initial instability region. Time $t=1204 T_{0}$. 


\section{Instability of the standing wave.}

Maybe the most practically important case of surface wave instability is the case of instability of the standing wave when we have interaction of two waves $a_{\mathbf{k}_{0}}$ and $a_{-\mathbf{k}_{0}}$. In this case, the resonant curve is a circle with the center at zero $\mathbf{k}=\mathbf{0}$ and of radius $\left|\mathbf{k}_{0}\right|$. It is clear that such a process is general for any isotropic dispersion. The theory for similar (although in some remote way) instability in plasma was developed in [34].

\subsection{Standing capillary wave.}

Simulation results for the standing capillary $(\mu=0.1)$ wave are represented in Figures 25:29. Contour plots correspond to the level $\left|a_{\mathbf{k}}\right|^{2}=10^{-23}$, which is an order of magnitude higher than the background noise. The full picture of the

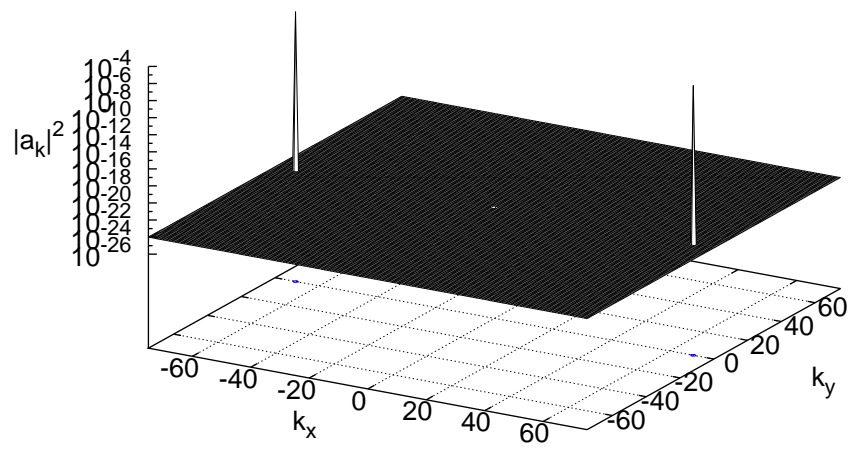

Figure 24: Instability of the standing capillary wave. Initial conditions. Time $t=0$.
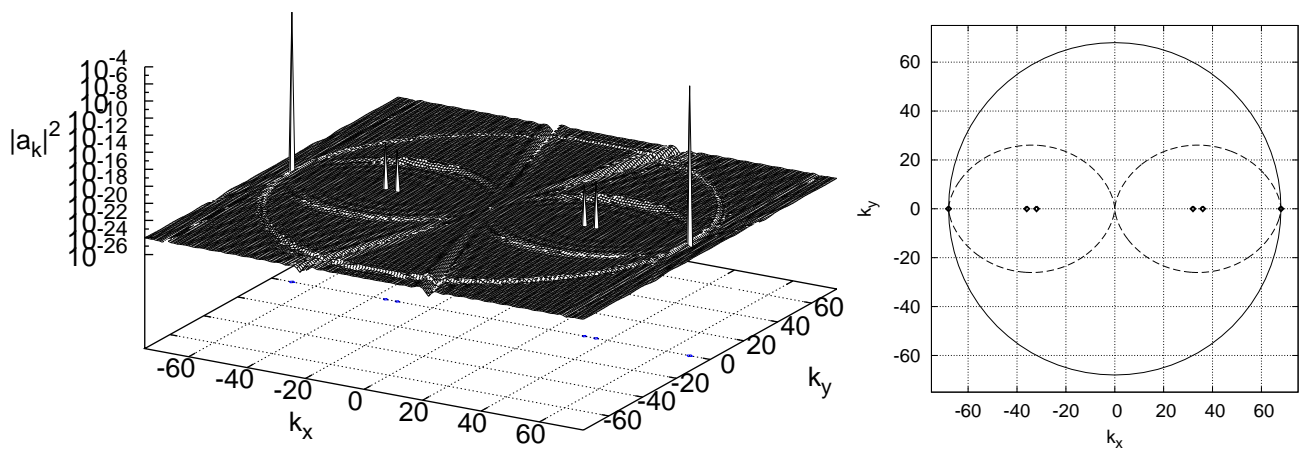

Figure 25: Instability of the standing capillary wave. Beginning of growth on the resonant curves. Some nonresonant absorption is noticeable. Time $t=14 T_{0}$.

$k$-plane in the final moment of simulations is represented in Figure 30, A closer 26 

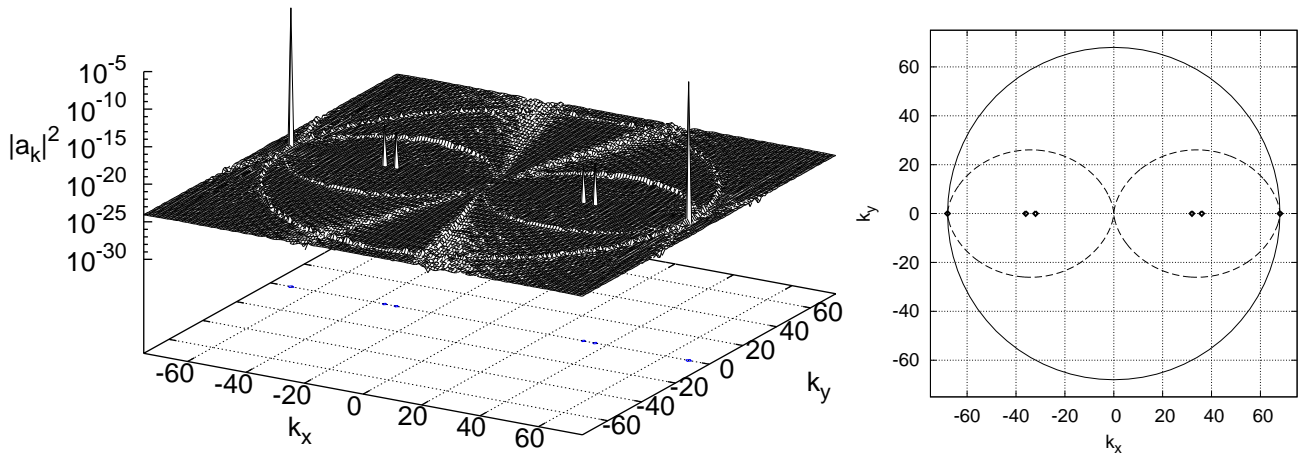

Figure 26: Instability of the standing capillary wave. Growth on the resonant curves continues. Time $t=57 T_{0}$.
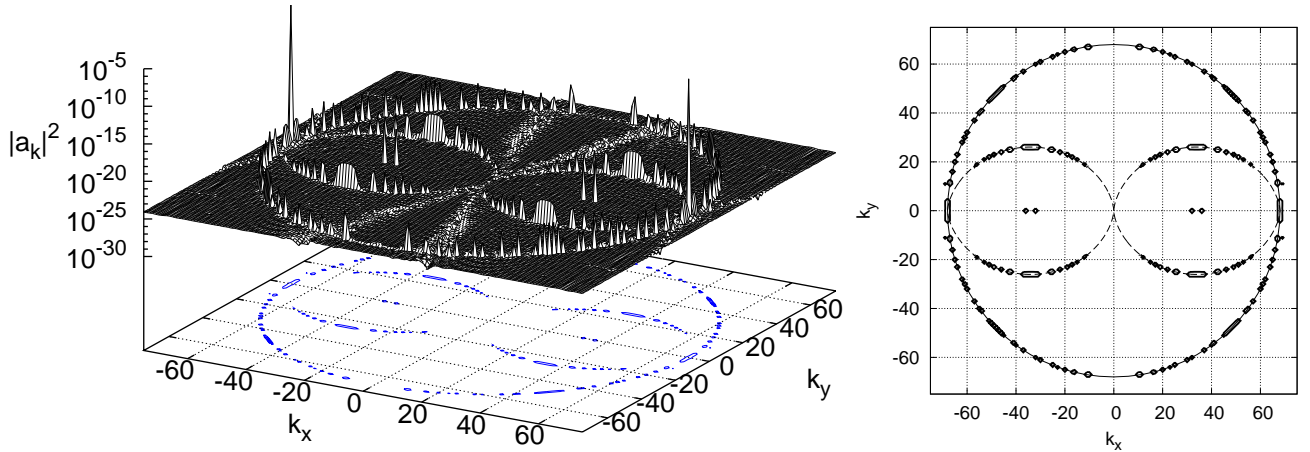

Figure 27: Instability of the standing capillary wave. Unstable harmonics are well developed. Time $t=283 T_{0}$.
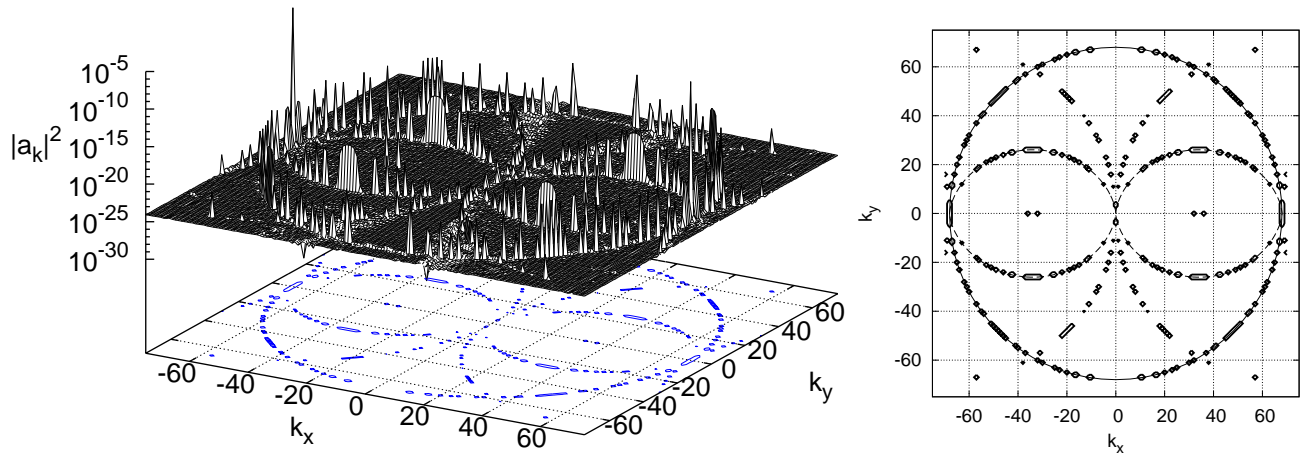

Figure 28: Instability of the standing capillary wave. One can notice the formation of the forced harmonics as copies of the initial circle shifted by $\pm \mathbf{k}_{0}$ vectors. Time $t=509 T_{0}$. 

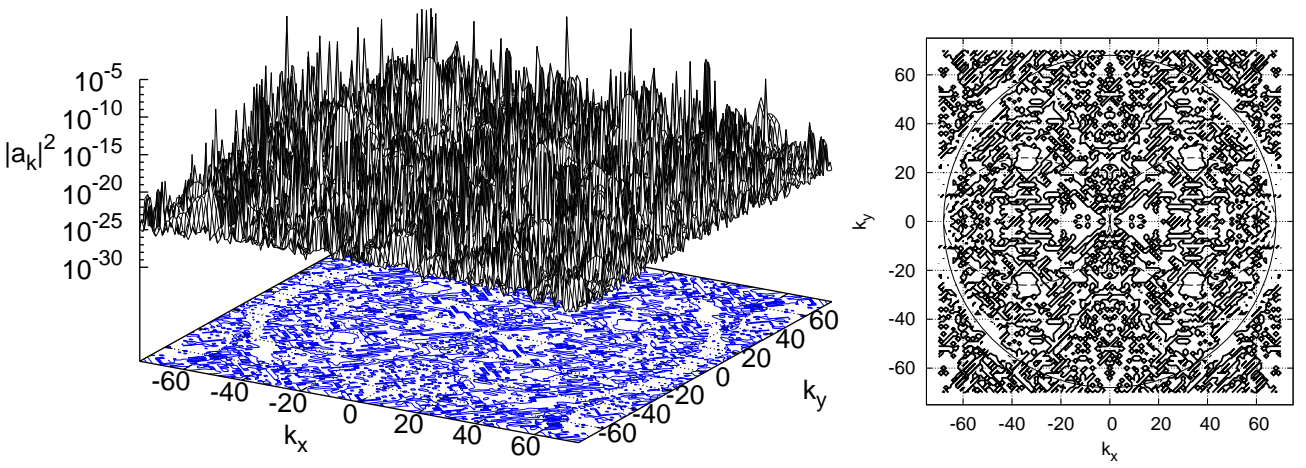

Figure 29: Instability of the standing capillary wave. Isotropization started. The plane is filled by secondary decay processes. Time $t=1018 T_{0}$.

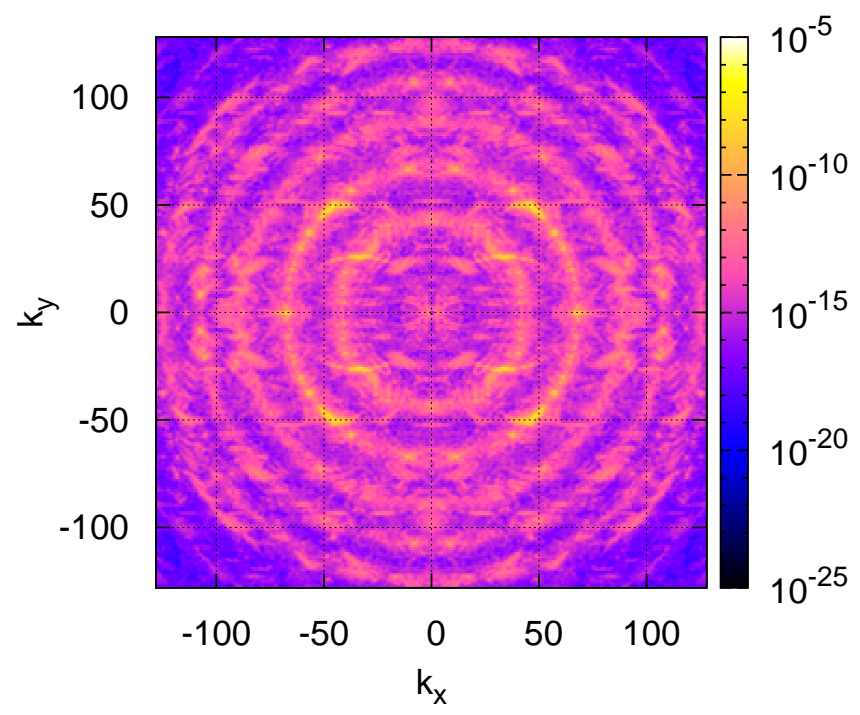

Figure 30: Instability of the standing capillary wave. Full $k$-plane. Time $t=2587 T 0$. 
snapshot of the most interesting region of Figure 30 (initial instability region) is represented in Figure 31, We observe isotropization of the wave field, but in

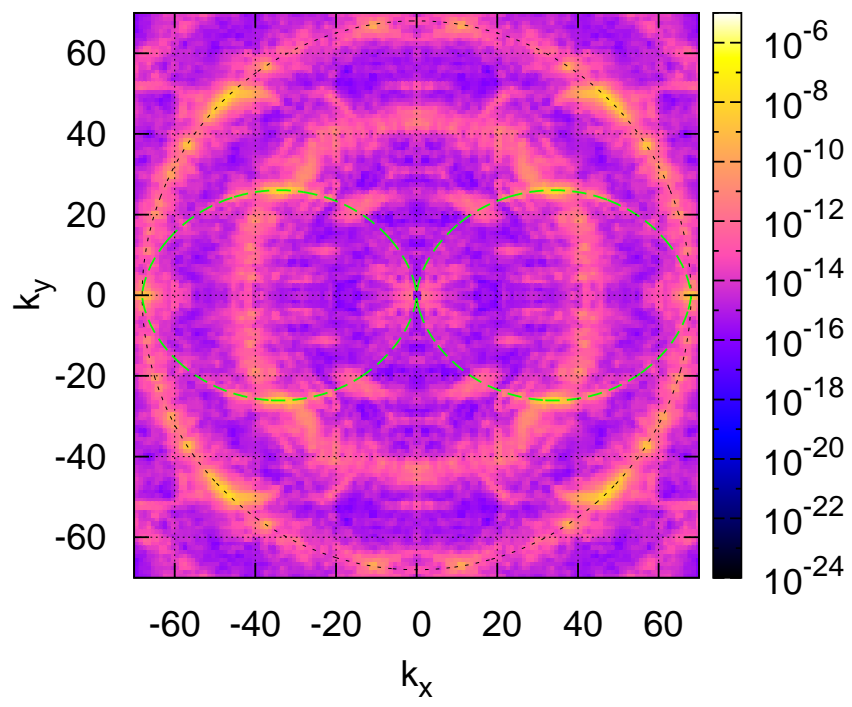

Figure 31: Instability of the standing capillary wave. A closer snapshot of the initial instability region. The inner circle corresponds to instability of the standing waves produced in the points of the maximum of the growth rate for decay of every individual initial wave. Compare position with the most developed harmonics inside the main circle in Figure 27 Time $t=2587 T 0$.

order to obtain a smooth spectrum we need to wait much longer. 


\subsection{Standing gravity wave.}

Simulation results for the standing gravity wave of steepness $\mu=0.1$ are represented in Figures 33 37. Contour plots correspond to the level $\left|a_{\mathbf{k}}\right|^{2}=10^{-23}$, which is an order of magnitude higher than the background noise. The

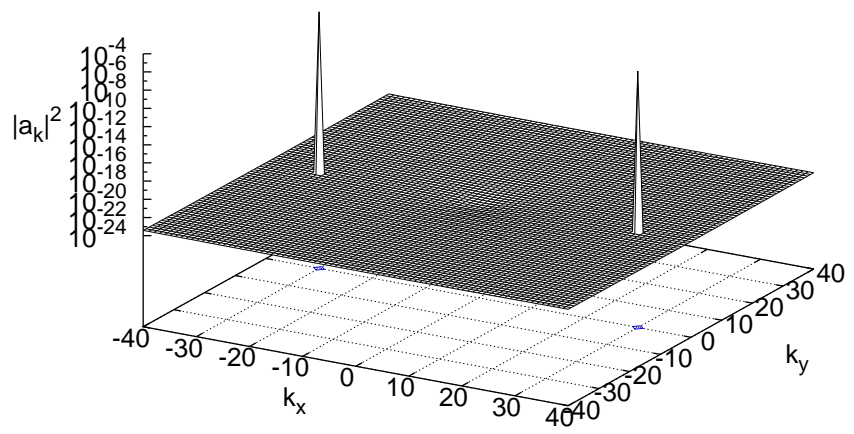

Figure 32: Instability of the standing gravity wave. Initial conditions. Time $t=0$.
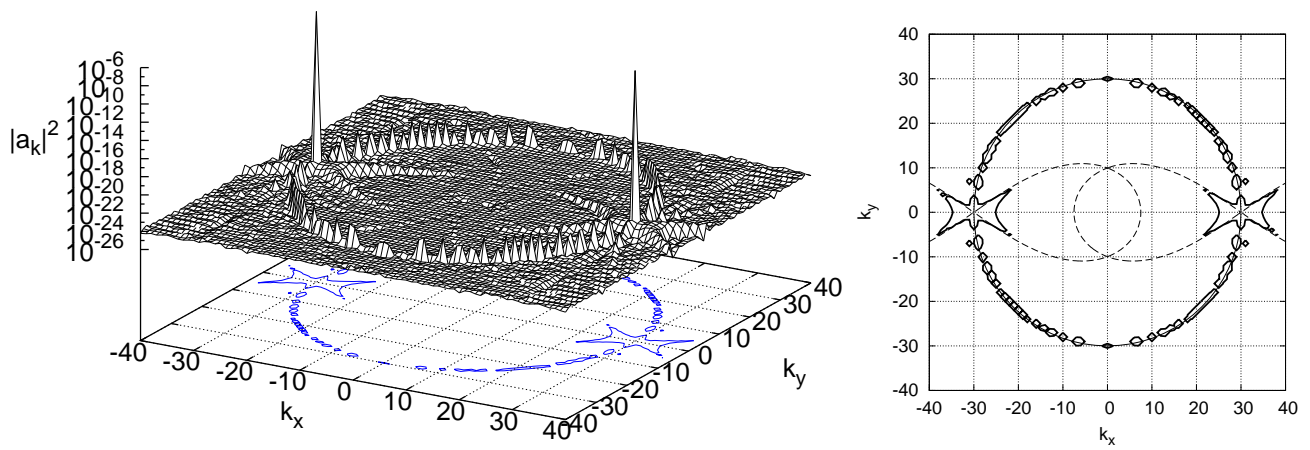

Figure 33: Instability of the standing gravity wave. Unstable harmonics on the resonant curves begin to grow. Time $t=116 T_{0}$.

full picture of the $k$-plane in the final moment of simulations is represented in Figure 38, A closer look at the most interesting region of Figure 38 (initial instability region) is represented in Figure 39. Finally, we observe almost complete isotropization of the wave field, although we started from just two waves. Weak angle dependence resembles the $\cos (\theta)$ of coupling coefficient (51). The observed process can be used for the generation of an isotropic wave field through initial generation of the standing wave, which in turn through the discussed instability will generate an isotropic spectrum. This is quite a nontrivial problem for direct wave generation. 

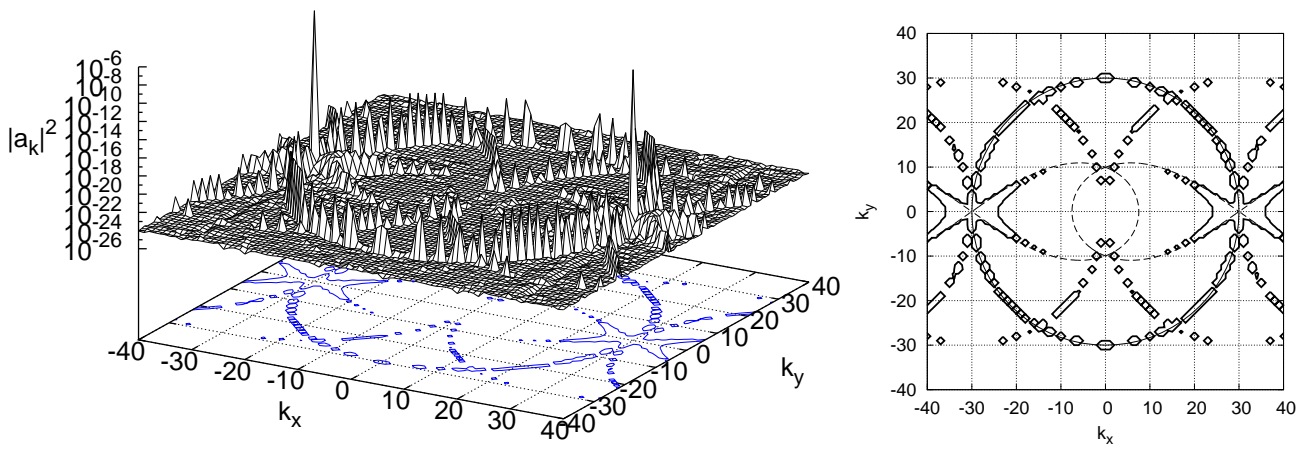

Figure 34: Instability of the standing gravity wave. Unstable harmonics are well developed. Time $t=232 T_{0}$.
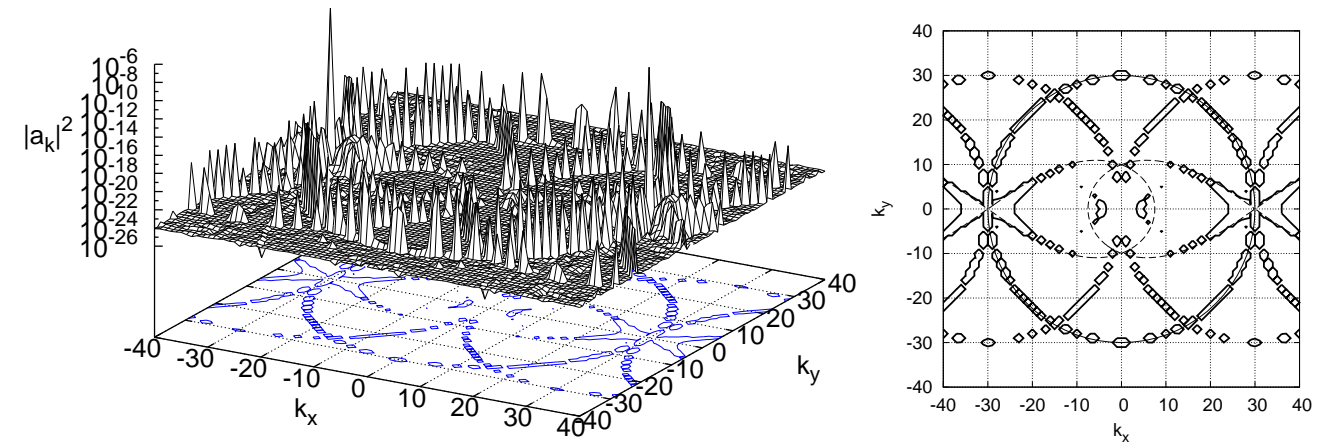

Figure 35: Instability of the standing gravity wave. Formation of forced harmonics corresponding to initial circle shifter with $\pm \mathbf{k}_{0}$ vectors. Time $t=348 T_{0}$.
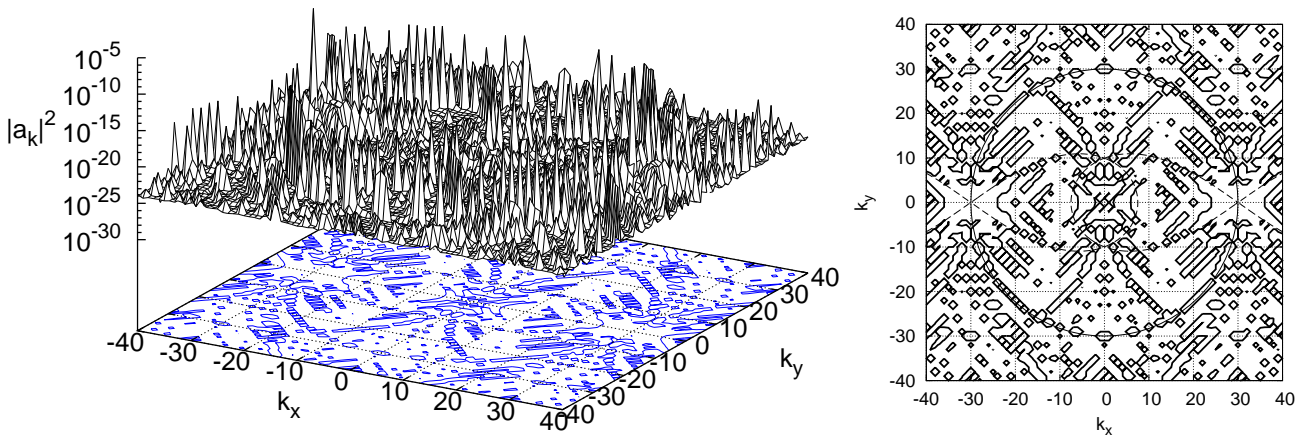

Figure 36: Instability of the standing gravity wave. Secondary processes reveal themselves. Time $t=463 T_{0}$. 

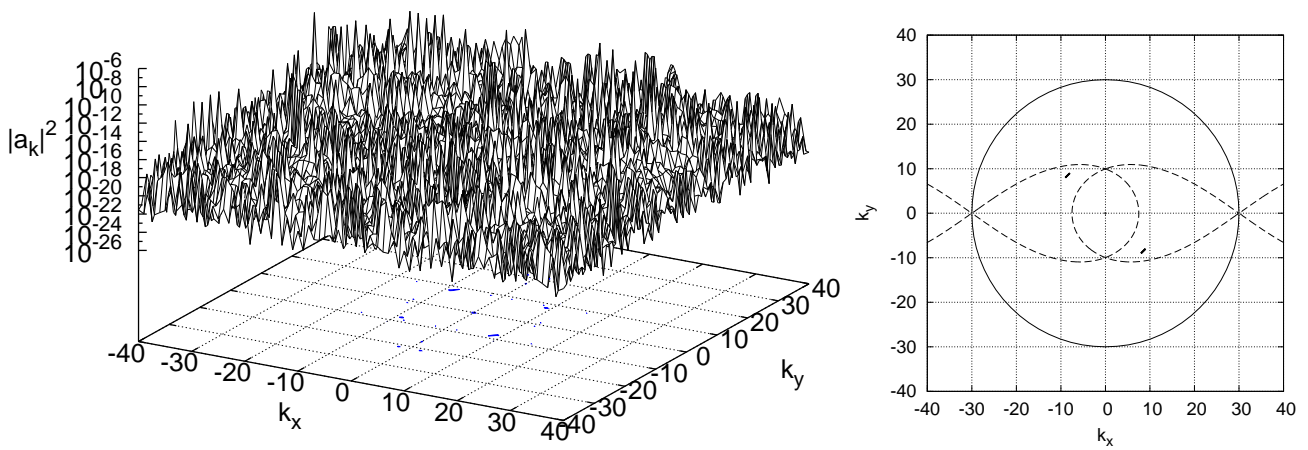

Figure 37: Instability of the standing gravity wave. Stochastization of the wave field begins. Time $t=580 T_{0}$.

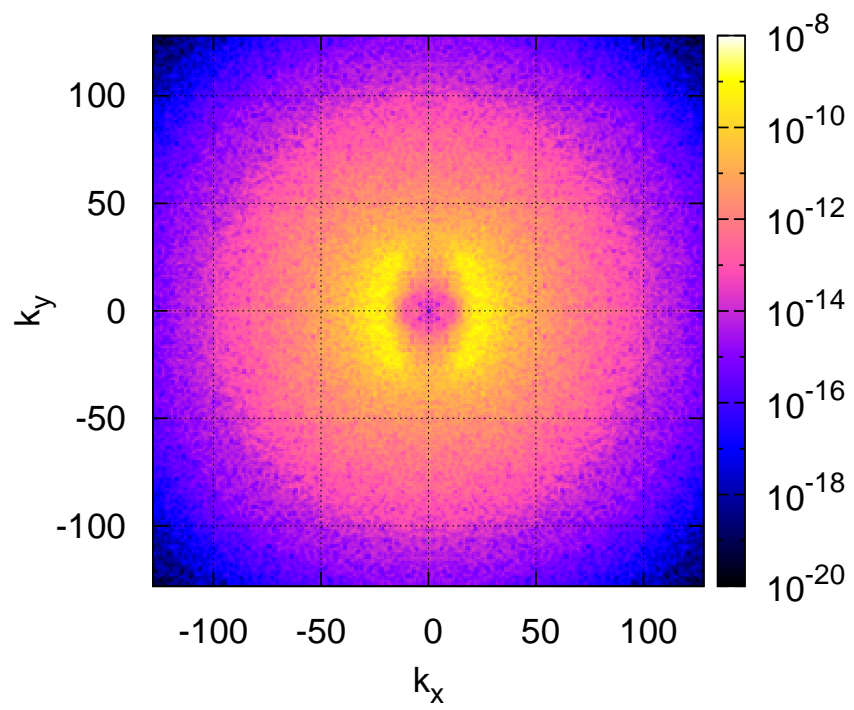

Figure 38: Instability of the standing gravity wave. Full $k$-plane. Time $t=3068 T_{0}$. 


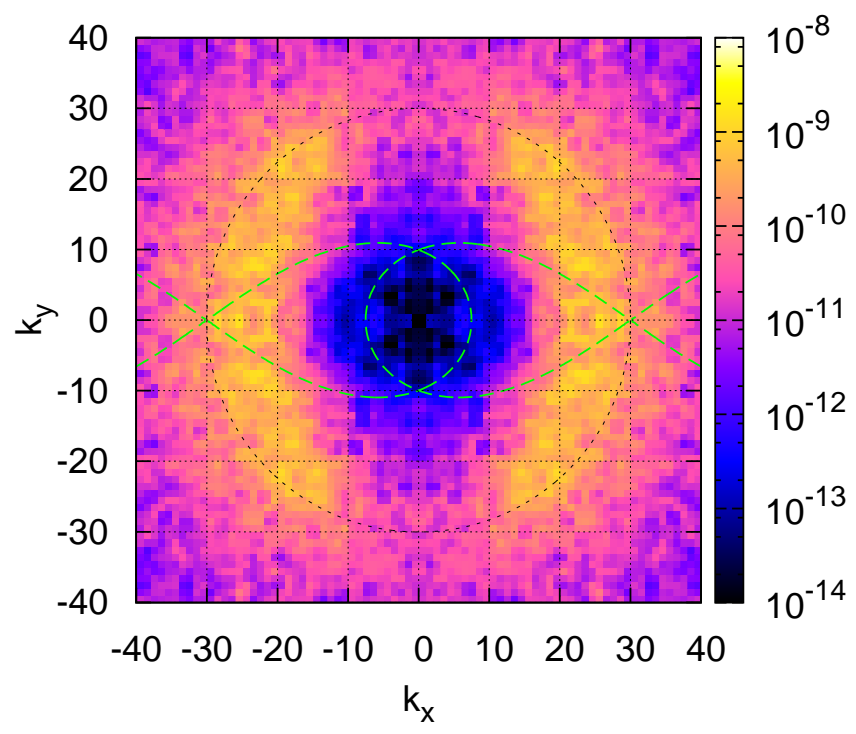

Figure 39: Instability of the standing gravity wave. A closer look at the initial instability region. Time $t=3068 T_{0}$.

In our simulation, we observed start of formation of the weakly turbulent spectrum tail (see Figure 40) and formation of Kolmogorov-Zakharov weak turbulent spectrum of direct cascade [31].

In conclusion, we have to note that although the wave amplitudes should be high enough to make grid discreteness unimportant, at the same time they must be low enough to satisfy weak nonlinearity conditions. 


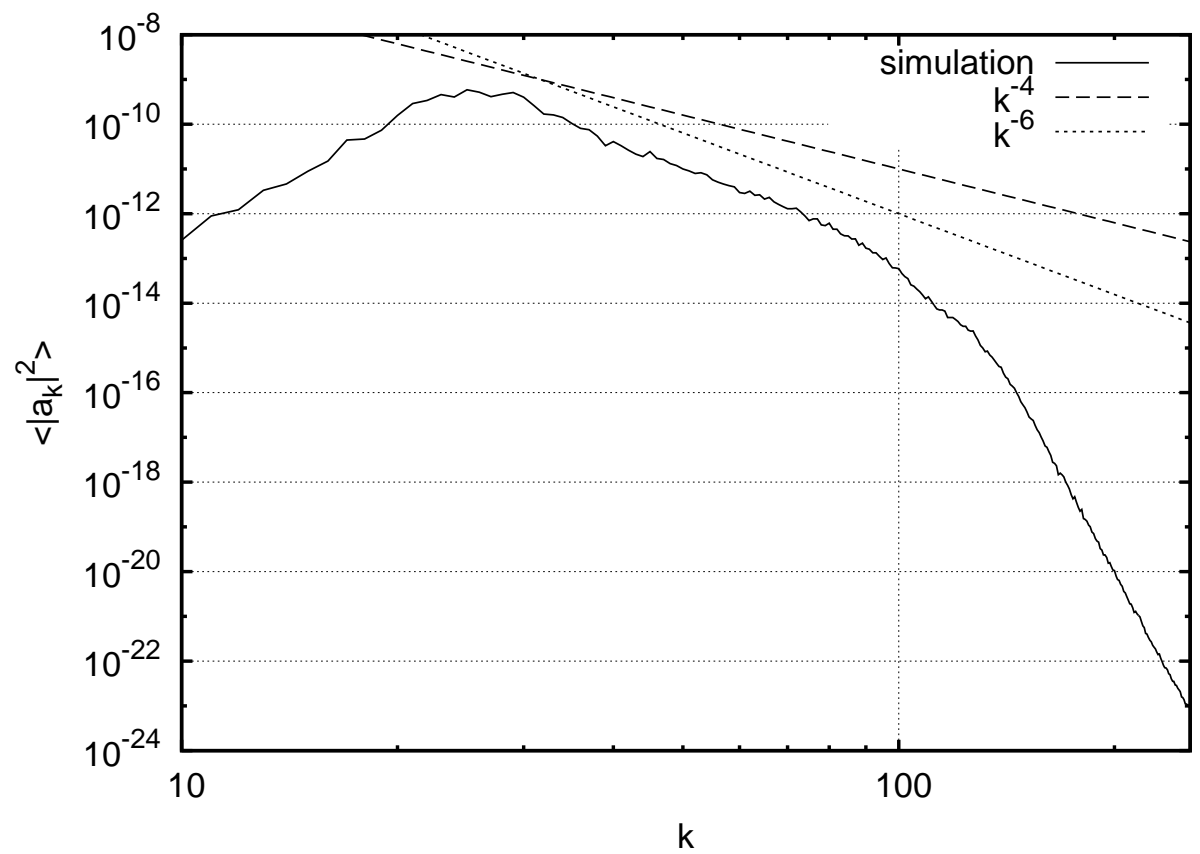

Figure 40: Instability of the standing gravity wave. Beginning of the formation of the weakly turbulent spectrum tail. Solid line: angle averaged spectrum from simulation results; dashed line: theoretically predicted KZ-spectrum [31]; dotted line: the best fit with power-like function. Time $t=3068 T_{0}$. 


\section{Conclusion.}

We gave a complete theoretical description of the three- and four-wave instabilities due to the resonant interactions of waves. In order to simulate wave turbulence, these mechanisms have to work, even on homogeneous grid where the exact resonance conditions are never fulfilled. We demonstrated the possibility to achieve resonant interactions on homogeneous grid in numerical simulations. Simulation results are in good agreement with the theoretical predictions. Stochastization of the capillary wave field was recently observed in the laboratory experiment [35]. It is interesting to note, that formation of the spectrum tail corresponding to the flux of energy was obtained only over some threshold value of pumping force. This is in good agreement with our estimations: only after waves reach some critical amplitude, discreteness of the wavenumber grid become negligible. Numerical observation and some theoretical estimations for similar behavior of gravity waves are given in [21].

Also, we described in details the algorithm for the simulation of weakly nonlinear gravity-capillary surface waves. The numerical scheme, which was used in the code, conserves Hamiltonian of the system. Features of the algorithm are used to conveniently control the adaptive time step. The described pseudospectral method allowed us to simulate wave turbulence in numerous cases.

We discussed and simulated the instability of standing wave for both capillary and gravity waves. Numerical simulations show that the instability of propagating waves leads to the formation of anisotropic, weakly-turbulent spectra while the instability of standing waves leads to the generation of almost isotropic spectra, demonstrating the tendency to formation of Kolmogorov-Zakharov tails. We conclude that the numerical simulation of wave instability is a perfect tool for the study of Wave Turbulence Theory. For experimental wave tanks, this instability provides a very simple and robust approach, which allows the production of isotropic wave fields through excitation of just one standing wave.

Current experimental science in the field of surface waves is on the rise. Recent advances allow to perform such state of the art experiments, like formation of the "tractor beam" using the propagating surface waves [36]. For observation of the standing wave circular instability the ability to reconstruct wavenumbers spectrum is crucial. Currently authors interact with one of the group of experimentalists in order to perform an experiment. An idea is very simple: using parametric excitation generate a standing wave in the rectangular cell and then follow the change of the wavenumbers spectrum. What is interesting, parametric excitation of waves is a standard technique in surface waves laboratory experiments (e.g. see [35]), so essentially even processing of the already existing data 
would be sufficient. The preliminary results are very promising. A complete report will be published separately.

This paper as a whole can be used as a comprehensive guide for theoretical and computational approaches to simulation of weakly nonlinear waves on the surface of fluids.

\section{Acknowledgments.}

The authors gratefully wish to acknowledge the following contributions:

KAO was supported by the National Science Foundation grant OCE 1131791, and during the summer visit partially supported by the grant NSh-6885.2010.2. The condition for iterations stability and Appendix C were derived with support of the Russian Scientific Foundation grant 14-22-00259.

ZVE was partially supported by the NSF grant 1130450 .

Both DAI and ZVE were supported by the Russian Scientific Foundation grant 14-22-00174. In particular, with this support growth rates for four- and three-wave interactions were calculated.

Also authors would like to thank developers of FFTW [37] and the whole GNU project [38] for developing, and supporting this useful and free software.

\section{Appendix A. Discrete Hamiltonian variation.}

Let us derive a variation of Hamiltonian $\left(H^{n+1}-H^{n}\right)$. The $\hat{k}$ operator is selfadjoined

$$
\int g \hat{k} f \mathrm{~d}^{2} r=\int f \hat{k} g \mathrm{~d}^{2} r .
$$

Let us perform a variation in detail for the quadratic part of the Hamiltonian (12) in the case of surface gravity waves.

$$
H_{0}=\frac{1}{2} \int\left(\psi \hat{k} \psi+g \eta^{2}\right) \mathrm{d}^{2} r
$$




$$
\begin{aligned}
& \Delta H_{0}=H_{0}^{n+1}-H_{0}^{n}= \\
& =\frac{1}{2} \int\left(\psi^{n+1} \hat{k} \psi^{n+1}-\psi^{n} \hat{k} \psi^{n}\right) \mathrm{d}^{2} r+\frac{g}{2} \int\left(\eta^{n+1^{2}}-\eta^{n 2}\right) \mathrm{d}^{2} r= \\
& =\frac{1}{2} \int\left(\psi^{n+1} \hat{k} \psi^{n+1}-\psi^{n} \hat{k} \psi^{n+1}+\psi^{n} \hat{k} \psi^{n+1}-\psi^{n} \hat{k} \psi^{n}\right) \mathrm{d}^{2} r+ \\
& \frac{g}{2} \int\left(\eta^{n+1}-\eta^{n}\right)\left(\eta^{n+1}+\eta^{n}\right) \mathrm{d}^{2} r=\frac{1}{2} \int\left[\left(\psi^{n+1}-\psi^{n}\right) \hat{k} \psi^{n+1}+\right. \\
& \left.+\psi^{n} \hat{k}\left(\psi^{n+1}-\psi^{n}\right)\right] \mathrm{d}^{2} r+\frac{g}{2} \int\left(\eta^{n+1}-\eta^{n}\right)\left(\eta^{n+1}+\eta^{n}\right) \mathrm{d}^{2} r= \\
& =\frac{1}{2} \int\left(\psi^{n+1}-\psi^{n}\right) \hat{k}\left(\psi^{n+1}+\psi^{n}\right) \mathrm{d}^{2} r+\frac{g}{2} \int\left(\eta^{n+1}-\eta^{n}\right)\left(\eta^{n+1}+\eta^{n}\right) \mathrm{d}^{2} r= \\
& =\frac{1}{2} \int \Delta \psi \hat{k}\left(\psi^{n+1}+\psi^{n}\right) \mathrm{d}^{2} r+\frac{g}{2} \int \Delta \eta\left(\eta^{n+1}+\eta^{n}\right) \mathrm{d}^{2} r=
\end{aligned}
$$

Here and further $\Delta \psi=\left(\psi^{n+1}-\psi^{n}\right)$ and $\Delta \eta=\left(\eta^{n+1}-\eta^{n}\right)$.

Similar calculations give us all other variations.

For short, let us omit integral signs in varied expressions.

Quadratic terms

$$
\begin{aligned}
& \Delta\left(\frac{1}{2} \int \psi \hat{k} \psi \mathrm{d}^{2} r\right) \rightarrow \frac{1}{2} \Delta \psi \hat{k}\left(\psi^{n+1}+\psi^{n}\right) ; \\
& \Delta\left(\frac{1}{2} \int \frac{\omega_{k}^{2}}{|\mathbf{k}|}\left|\eta_{\mathbf{k}}\right|^{2} \mathrm{~d} \mathbf{k}\right) \rightarrow \frac{1}{2} \Delta \eta_{\mathbf{k}} \frac{\omega_{k}^{2}}{|\mathbf{k}|}\left(\eta_{\mathbf{k}}^{n+1}+\eta_{\mathbf{k}}^{n}\right) .
\end{aligned}
$$

Cubic terms

$$
\begin{aligned}
\Delta\left(\frac{1}{2} \int \eta|\nabla \psi|^{2} \mathrm{~d}^{2} r\right) \longrightarrow & -\frac{1}{4} \Delta \psi\left(\nabla,\left(\eta^{n+1}+\eta^{n}\right) \nabla\left(\psi^{n+1}+\psi^{n}\right)\right)+ \\
& +\frac{1}{4} \Delta \eta\left(\left|\nabla \psi^{n+1}\right|^{2}+\left|\nabla \psi^{n}\right|^{2}\right) \\
\Delta\left(\frac{1}{2} \int \eta(\hat{k} \psi)^{2} \mathrm{~d}^{2} r\right) \longrightarrow & \left.-\frac{1}{4} \Delta \psi \hat{k}\left(\eta^{n+1}+\eta^{n}\right) \hat{k}\left(\psi^{n+1}+\psi^{n}\right)\right)- \\
& -\frac{1}{4} \Delta \eta\left(\left(\hat{k} \psi^{n+1}\right)^{2}+\left(\hat{k} \psi^{n}\right)^{2}\right) .
\end{aligned}
$$

Quartic terms

$$
\begin{aligned}
\Delta\left(\frac{1}{2} \int(\eta \hat{k} \psi) \hat{k}(\eta \hat{k} \psi) \mathrm{d}^{2} r\right) \longrightarrow & \frac{1}{4} \Delta \psi \hat{k}\left[\left(\eta^{n+1}+\eta^{n}\right) \times\right. \\
& \left.\times \hat{k}\left(\eta^{n+1} \hat{k} \psi^{n+1}+\eta^{n} \hat{k} \psi^{n}\right)\right]+ \\
& +\frac{1}{4} \Delta \eta \hat{k}\left[\left(\psi^{n+1}+\psi^{n}\right) \times\right. \\
& \left.\times \hat{k}\left(\eta^{n+1} \hat{k} \psi^{n+1}+\eta^{n} \hat{k} \psi^{n}\right)\right]
\end{aligned}
$$




$$
\begin{aligned}
\Delta\left(\frac{1}{2} \int\left(\nabla^{2} \psi\right)(\hat{k} \psi) \eta^{2} \mathrm{~d}^{2} r\right) \longrightarrow & \frac{1}{8} \Delta \psi \nabla^{2}\left[\left(\left(\eta^{n+1}\right)^{2}+\left(\eta^{n}\right)^{2}\right) \times\right. \\
& \left.\times \hat{k}\left(\psi^{n+1}+\psi^{n}\right)\right]+ \\
& +\frac{1}{8} \Delta \psi \hat{k}\left[\left(\left(\eta^{n+1}\right)^{2}+\left(\eta^{n}\right)^{2}\right) \times\right. \\
& \left.\times \nabla^{2}\left(\psi^{n+1}+\psi^{n}\right)\right]+ \\
& +\frac{1}{4} \Delta \eta\left(\eta^{n+1}+\eta^{n}\right) \times \\
& \times\left(\nabla^{2} \psi^{n+1} \hat{k} \psi^{n+1}+\nabla^{2} \psi^{n} \hat{k} \psi^{n}\right) .
\end{aligned}
$$

\section{Appendix B. Matrix elements}

We repeat formulae from [28].

$$
\begin{aligned}
& V^{(1,2)}\left(\mathbf{k}, \mathbf{k}_{1}, \mathbf{k}_{2}\right)=\frac{1}{4 \pi \sqrt{2}}\left\{\left(\frac{A_{k} B_{k_{1}} B_{k_{2}}}{B_{k} A_{k_{1}} A_{k_{2}}}\right)^{1 / 4} L^{(1)}\left(\mathbf{k}_{1}, \mathbf{k}_{2}\right)\right. \\
& \left.\left.-\left(\frac{B_{k} A_{k_{1}} B_{k_{2}}}{A_{k} A_{k_{1}} A_{k_{2}}}\right)^{1 / 4} L^{(1)}\left(-\mathbf{k}, \mathbf{k}_{1}\right)-\left(\frac{B_{k} B_{k_{1}} A_{k_{2}}}{A_{k} A_{k_{1}} B_{k_{2}}}\right)^{1 / 4} L^{(1)}(-\mathbf{k}), \mathbf{k}_{2}\right)\right\} \\
& V^{(0,3)}\left(\mathbf{k}, \mathbf{k}_{1}, \mathbf{k}_{2}\right)=\frac{1}{4 \pi \sqrt{2}}\left\{\left(\frac{A_{k} B_{k_{1}} B_{k_{2}}}{B_{k} A_{k_{1}} A_{k_{2}}}\right)^{1 / 4} L^{(1)}\left(\mathbf{k}_{1}, \mathbf{k}_{2}\right)\right. \\
& \left.\left.+\left(\frac{B_{k} A_{k_{1}} B_{k_{2}}}{A_{k} B_{k_{1}} A_{k_{2}}}\right)^{1 / 4} L^{(1)}\left(\mathbf{k}, \mathbf{k}_{1}\right)+\left(\frac{B_{k} B_{k_{1}} A_{k_{2}}}{A_{k} A_{k_{1}} B_{k_{2}}}\right)^{1 / 4} L^{(1)}(\mathbf{k}), \mathbf{k}_{2}\right)\right\} \\
& A_{k}=|k|, \quad b_{k}=g+\sigma k^{2} . \\
& V_{\mathbf{k}, \mathbf{k}_{1}, \mathbf{k}_{2}, \mathbf{k}}^{(2,2)}=3 .
\end{aligned}
$$




$$
\begin{aligned}
& a_{\mathbf{k}}^{(0)}=b_{\mathbf{k}}, \\
& a_{\mathbf{k}}^{(1)}=\int \Gamma^{(1)}\left(\mathbf{k}, \mathbf{k}_{1}, \mathbf{k}_{2}\right) b_{\mathbf{k}_{1}} b_{\mathbf{k}_{2}} \delta\left(\mathbf{k}-\mathbf{k}_{1}-\mathbf{k}_{2}\right) \mathrm{d} \mathbf{k}_{1} \mathrm{~d} \mathbf{k}_{2} \\
& -2 \int \Gamma^{(1)}\left(\mathbf{k}_{2}, \mathbf{k}, \mathbf{k}_{1}\right) b_{\mathbf{k}_{1}}^{*} b_{\mathbf{k}_{2}} \delta\left(\mathbf{k}+\mathbf{k}_{2}-\mathbf{k}_{2}\right) \mathrm{d} \mathbf{k}_{1} \mathrm{~d} \mathbf{k}_{2} \\
& +\int \Gamma^{(2)}\left(\mathbf{k}, \mathbf{k}_{1}, \mathbf{k}_{2}\right) b_{\mathbf{k}_{1}}^{*} b_{\mathbf{k}_{2}}^{*} \delta\left(\mathbf{k}+\mathbf{k}_{1}+\mathbf{k}_{2}\right) \mathrm{d} \mathbf{k}_{1} \mathrm{~d} \mathbf{k}_{2} \\
& a_{\mathbf{k}}^{(2)}=\int B\left(\mathbf{k}, \mathbf{k}_{1}, \mathbf{k}_{2}, \mathbf{k}_{3}\right) b_{\mathbf{k}_{1}}^{*} b_{\mathbf{k}_{2}} b_{\mathbf{k}_{3}} \delta\left(\mathbf{k}-\mathbf{k}_{1}-\mathbf{k}_{2}-\mathbf{k}_{3}\right) \mathrm{d} \mathbf{k}_{1} \mathrm{~d} \mathbf{k}_{2} \mathrm{~d} \mathbf{k}_{3}+\cdots \\
& \Gamma^{(1)}\left(\mathbf{k}, \mathbf{k}_{1}, \mathbf{k}_{2}\right)=-\frac{1}{2} \frac{V^{(1,2)\left(\mathbf{k}_{1}, \mathbf{k}_{2}\right)}}{\omega_{k}-\omega_{k_{1}}-\omega_{k_{2}}} \\
& \Gamma^{(2)}\left(\mathbf{k}, \mathbf{k}_{1}, \mathbf{k}_{2}\right)=-\frac{1}{2} \frac{V^{(0,3)\left(\mathbf{k}_{1}, \mathbf{k}_{2}\right)}}{\omega_{k}-\omega_{k_{1}}-\omega_{k_{2}}} \\
& B\left(\mathbf{k}, \mathbf{k}_{1}, \mathbf{k}_{2}, \mathbf{k}_{3}\right)=\Gamma^{(1)}\left(\mathbf{k}_{1}, \mathbf{k}_{2}, \mathbf{k}_{1}-\mathbf{k}_{2}\right) \Gamma^{(1)}\left(\mathbf{k}_{3}, \mathbf{k}, \mathbf{k}_{3}-\mathbf{k}\right) \\
& +\Gamma^{(1)}\left(\mathbf{k}_{1}, \mathbf{k}_{3}, \mathbf{k}-\mathbf{k}_{3}\right) \Gamma^{(1)}\left(\mathbf{k}_{2}, \mathbf{k}, \mathbf{k}_{2}-\mathbf{k}\right) \\
& -\Gamma^{(1)}\left(\mathbf{k}, \mathbf{k}_{2}, \mathbf{k}-\mathbf{k}_{2}\right) \Gamma^{(1)}\left(\mathbf{k}_{3}, \mathbf{k}_{1}, \mathbf{k}_{3}-\mathbf{k}_{1}\right) \\
& -\Gamma^{(1)}\left(\mathbf{k}_{1}, \mathbf{k}_{3}, \mathbf{k}_{1}-\mathbf{k}_{3}\right) \Gamma^{(1)}\left(\mathbf{k}_{2}, \mathbf{k}_{1}, \mathbf{k}_{2}-\mathbf{k}_{1}\right) \\
& -\Gamma^{(1)}\left(\mathbf{k}+\mathbf{k}_{1}, \mathbf{k}, \mathbf{k}_{1}\right) \Gamma^{(1)}\left(\mathbf{k}_{2}+\mathbf{k}_{3}, \mathbf{k}, \mathbf{k}_{1}\right) \\
& +\Gamma^{(2)}\left(-\mathbf{k}-\mathbf{k}_{1}, \mathbf{k}, \mathbf{k}_{1}\right) \Gamma^{(2)}\left(-\mathbf{k}_{2}-\mathbf{k}_{3}, \mathbf{k}_{2}, \mathbf{k}_{3}\right)
\end{aligned}
$$




$$
\begin{aligned}
& T_{1234}=\frac{1}{2}\left(\tilde{T}_{1234}+\tilde{T}_{2134}\right) \\
& \tilde{T}_{1234}=-\frac{1}{16 \pi^{2}} \frac{1}{\left(k_{1} k_{2} k_{3} k_{4}\right)^{1 / 4}} \\
& \times\left\{-12 k_{1} k_{2} k_{3} k_{4}-2\left(\omega_{1}+\omega_{2}\right)^{2}\left[\omega_{3} \omega_{4}\left(\left(\mathbf{k}_{1} \cdot \mathbf{k}_{2}\right)-k_{1} k_{2}\right)\right.\right. \\
& \left.+\omega_{1} \omega_{2}\left(\left(\mathbf{k}_{3} \cdot \mathbf{k}_{4}\right)-k_{3} k_{4}\right)\right] \frac{1}{g^{2}} \\
& -2\left(\omega_{1}-\omega_{3}\right)^{2}\left[\omega_{2} \omega_{4}\left(\left(\mathbf{k}_{1} \cdot \mathbf{k}_{3}\right)+k_{1} k_{3}\right)+\omega_{1} \omega_{3}\left(\left(\mathbf{k}_{2} \cdot \mathbf{k}_{4}\right)+k_{2} k_{4}\right)\right] \frac{1}{g^{2}} \\
& -2\left(\omega_{1}-\omega_{4}\right)^{2}\left[\omega_{2} \omega_{3}\left(\left(\mathbf{k}_{1} \cdot \mathbf{k}_{4}\right)+k_{1} k_{4}\right)+\omega_{1} \omega_{4}\left(\left(\mathbf{k}_{2} \cdot \mathbf{k}_{3}\right)+k_{2} k_{3}\right)\right] \frac{1}{g^{2}} \\
& +\left[\left(\mathbf{k}_{1} \cdot \mathbf{k}_{2}\right)+k_{1} k_{2}\right]\left[\left(\mathbf{k}_{3} \cdot \mathbf{k}_{4}\right)+k_{3} k_{4}\right] \\
& +\left[-\left(\mathbf{k}_{1} \cdot \mathbf{k}_{3}\right)+k_{1} k_{3}\right]\left[-\left(\mathbf{k}_{2} \cdot \mathbf{k}_{4}\right)+k_{2} k_{4}\right] \\
& +\left[-\left(\mathbf{k}_{1} \cdot \mathbf{k}_{4}\right)+k_{1} k_{4}\right]\left[-\left(\mathbf{k}_{2} \cdot \mathbf{k}_{3}\right)+k_{2} k_{3}\right] \\
& +4\left(\omega_{1}+\omega_{2}\right)^{2} \frac{\left[\left(\mathbf{k}_{1} \cdot \mathbf{k}_{2}\right)-k_{1} k_{2}\right]\left[-\left(\mathbf{k}_{3} \cdot \mathbf{k}_{4}\right)-k_{3} k_{4}\right]}{\omega_{1+2}-\left(\omega_{1}+\omega_{2}\right)^{2}} \\
& +4\left(\omega_{1}-\omega_{3}\right)^{2} \frac{\left[\left(\mathbf{k}_{1} \cdot \mathbf{k}_{3}\right)+k_{1} k_{3}\right]\left[\left(\mathbf{k}_{2} \cdot \mathbf{k}_{4}\right)+k_{2} k_{4}\right]}{\omega_{1-3}-\left(\omega_{1}-\omega_{3}\right)^{2}} \\
& +4\left(\omega_{1}-\omega_{4}\right)^{2} \frac{\left[\left(\mathbf{k}_{1} \cdot \mathbf{k}_{4}\right)+k_{1} k_{4}\right]\left[\left(\mathbf{k}_{2} \cdot \mathbf{k}_{3}\right)+k_{2} k_{3}\right]}{\omega_{1-4}-\left(\omega_{1}-\omega_{4}\right)^{2}} .
\end{aligned}
$$

\section{Appendix C. On the stability of the weakly nonlinear free surface hydro- dynamic model}

In detail, this question was considered in [39], but here we shall follow the original consideration which was done by A. I. Dyachenko in 1995 (result was mentioned in [40]) with some changes for 3D hydrodynamics.

Let us consider a large scale solution $\left(\eta_{0}, \psi_{0}\right)$ of (18) with small scale perturbations $(\delta \eta, \delta \psi)$

$$
\eta=\eta_{0}+\delta \eta, \quad \psi=\psi_{0}+\delta \psi
$$

After the substitution of (C.1) into (18) we perform linearization with respect to small amplitude perturbations. In order to do this, we take into account difference of scales, which results in "frozen coefficients" (terms with $\eta_{0}$ and $\psi_{0}$ can be factored out from the expressions with operator $\hat{k}$ and derivatives). Also, we keep only first nonlinear terms in (18), which originate from cubic or three-wave 
terms in Hamiltonian (16). All these steps yield the following dispersion relation for perturbations:

$$
\left(\omega_{k}-\mathbf{v}_{0} \mathbf{k}\right)^{2}=\omega_{0 k}^{2}-\left(\hat{k} \psi_{0}\right)^{2} k^{2}
$$

here we introduced $\mathbf{v}_{0}=\nabla \psi_{0}$, which is similar to the velocity on the surface of the fluid and $\omega_{0 k}^{2}=\sigma k^{3}+g k$ which is the squared linear dispersion relation for gravity-capillary waves on the surface of fluids with infinite depth. Correction $\mathbf{v}_{0} \mathbf{k}$ on the left hand side is nothing else but Doppler frequency shift. As one can see, for small enough scales (large enough $k$-s), the second term on the right hand side of (C.2) will prevail resulting in a non-zero imaginary part for $\omega_{k}$. This means that we have small scale (or high frequency) instability.

If we consider the cubic terms in (18), which originate from quartic or fourwave terms in Hamiltonian (16), the equation for perturbations (keeping only the first nonlinear terms) yields:

$$
\left(\omega_{k}-\mathbf{v}_{0} \mathbf{k}\right)^{2}=\omega_{0 k}^{2}+\left(\hat{k} \psi_{0}\right)\left(\nabla^{2} \psi_{0}\right) k
$$

As one can see, the dispersion relation (C.3) has no instability, at least in the first order of nonlinearity. It means that even in the case of capillary waves, where three-wave interactions are the major process, the presence of terms corresponding to four-wave processes stabilizes the equation. If we consider the full expression for dispersion relation resulting from (18) with cubic terms:

$$
\begin{aligned}
\left(\omega_{k}-\mathbf{v}_{0} \mathbf{k}\right)^{2}= & \omega_{0 k}^{2}+\left(\hat{k} \psi_{0}\right)\left(\nabla^{2} \psi_{0}\right) k+2\left(\hat{k} \psi_{0}\right)\left(\hat{k}\left[\eta_{0} \hat{k} \psi_{0}\right]+\eta_{0} \nabla^{2} \psi_{0}\right) k^{2} \\
& -\left\{\left(\hat{k}\left[\eta_{0} \hat{k} \psi_{0}\right]\right)^{2}+2 \hat{k}\left[\eta_{0} \hat{k} \psi_{0}\right]\left(\eta_{0} \nabla^{2} \psi_{0}\right)+\left(\eta_{0} \nabla^{2} \psi_{0}\right)^{2}\right\} k^{2},
\end{aligned}
$$

one can see, that instability reappears only with fourth-order nonlinearity terms, which means that it can influence only computations with relatively high steepness or with very high values of $k$. This instability can be eliminated by proper canonical change of variables, as shown in [39].

\section{References}

[1] L. Debnath, Nonlinear water waves, Academic Press, 1994.

[2] L. N. Sretenskii, Theory of wave motion of fluid, ONTI, Moscow, 1936.

[3] A. Nekrasov, On steady waves, Izv. Ivanovo-Voznesensk. Polytech. Inst. 3 (1921) 52-56.

[4] I. R. Young, The exact theory of standing waves on the surface of heavy fluid, Izdat. Akad. Nauk. SSSR, Moscow, 1951.

[5] T. Levi-Civita, Determination rigoureuse des ondes permanentes d'ampleur finie, Math. Ann. 93 (1925) 264-314. 
[6] S. A. Dyachenko, P. M. Lushnikov, A. O. Korotkevich, The complex singularity of a stokes wave, JETP Letters 98 (11) (2013) 767-771.

[7] S. A. Dyachenko, P. M. Lushnikov, A. O. Korotkevich, Branch cuts of Stokes wave on deep water. Part I: Numerical solution and Padé approximation, Submitted to Studies in Applied MathematicsarXiv: 1507.02784

[8] P. M. Lushnikov, Branch cuts of Stokes wave on deep water. Part II: Structure and location of branch points in infinite set of sheets of Riemann surface, Submitted to Journal of Fluid MechanicsarXiv: 1509.03393

[9] M. J. Lighthill, Contribution to the theory of waves in nonlinear dispersive systems, J. Inst. Maths. Applics. 1 (1965) 269-306.

[10] V. E. Zakharov, The instability of waves in nonlinear dispersive media, J. Exp. Teor. Phys. 24 (4) (1967) 740-744.

[11] T. Benjamin, Instability of periodic wave trains in nonlinear dispersive systems, Proc. Roy. Soc. London A.299 (1967) 59-75.

[12] T. Benjamin, J. Feir, The disintegration of wave trains on deep water. part 1. theory, J. Fluid Mech. 27 (1967) 417-430.

[13] V. E. Zakharov, Stability of periodic waves of finite amplitude on a surface, J. Appl. Mech. Tech. Phys. 9 (2) (1968) 190-194.

[14] V. E. Zakharov, L. A. Ostrovsky, Modulational instability: The beginning, Physica D 238 (2005) 540-548.

[15] Y. V. Sedletsky, The hamiltonian formalism and a new type of modulation instability, J. Phys. A: Math. Theor. 41 (2008) 035502.

[16] S. A. Dyachenko, D. V. Zakharov, V. E. Zakharov, Non-periodic one-gap potentials in quantum mechanicsarXiv:1505.05806

[17] V. E. Zakharov, V. S. Lvov, G. Falkovich, Kolmogorov Spectra of Turbulence I, SpringerVerlag, Berlin, 1992.

[18] A. I. Dyachenko, A. O. Korotkevich, V. E. Zakharov, Decay of the monochromatic capillary wave, JETP Lett. 77 (9) (2003) 477-481.

[19] A. I. Dyachenko, A. O. Korotkevich, V. E. Zakharov, Weak turbulence of gravity waves, JETP Lett. 77 (10) (2003) 546-550.

[20] A. I. Dyachenko, A. O. Korotkevich, V. E. Zakharov, Weak turbulent kolmogorov spectrum for surface gravity waves, Phys. Rev. Lett. 92 (13) (2004) 134501.

[21] V. E. Zakharov, A. O. Korotkevich, A. Pushkarev, A. I. Dyachenko, Mesoscopic wave turbulence, JETP Lett. 82 (8) (2005) 487-491.

[22] V. E. Zakharov, A. O. Korotkevich, A. Pushkarev, D. Resio, Coexistence of weak and strong wave turbulence in a swell propagation, Phys. Rev. Lett. 99 (16) (2007) 164501.

[23] A. O. Korotkevich, A. Pushkarev, D. Resio, V. E. Zakharov, Numerical verification of the weak turbulent model for swell evolution, Eur. J. Mech. B/Fluids 27 (4) (2008) 361-387.

[24] A. O. Korotkevich, Simultaneous numerical simulation of direct and inverse cascades in wave turbulence, Phys. Rev. Lett. 101 (7) (2008) 074504.

[25] A. O. Korotkevich, Influence of the condensate and inverse cascade on the direct cascade in wave turbulence, Math. Comput. Simul. 82 (7) (2012) 1228-1238.

[26] P. Saffman, H. Yuen, Three dimensional waves on deep water, Advances in Nonlinear Waves 2 (1985) 1-30.

[27] V. Krasitskii, On the canonical transformation of theory of weakly nonlinear waves with nondecay spectral law, Sov. Phys. JETP 98 (1990) 1644-1655. 
[28] V. E. Zakharov, Statistical theory of gravity and capillary waves on the surface of a finitedepth fluid, Eur. J. Mech. B/Fluids 18 (3) (1999) 327-344.

[29] O. M. Phillips, Theoretical and experimental studies of gravity waves interactions, Proc. Roy. Soc. London A.299 (1967) 104-119.

[30] A. I. Dyachenko, V. E. Zakharov, Is free surface hydrodynamics an integrable system?, Phys. Lett. A 190 (2) (1994) 144-148.

[31] V. E. Zakharov, N. N. Filonenko, Energy spectrum for stochastic oscillations of the surface of a liquid, Sov. Phys. Dokl. 11 (1967) 881-884.

[32] A. I. Dyachenko, A. C. Newell, A. Pushkarev, V. E. Zakharov, Optical turbulence: weak turbulence, condensates and collapsing fragments in the nonlinear schroedinger equation, Physica D 57 (1992) 96-160.

[33] T. Sauer, Numerical Analysis, 2nd Edition, Pearson, 2011.

[34] A. Berkhoer, V. E. Zakharov, Self-excitation of waves with different polarization in nonlinear media, J. Exp. Theor. Phys. 31 (3) (1970) 486-490.

[35] H. Xia, M. Shats, H. Punzmann, Modulation instability and capillary wave turbulence, Europhys. Lett. 91 (2010) 14002.

[36] H. Punzmann, N. Francois, H. Xia, G. Falkovich, M. Shats, Generation and reversal of surface flows by propagating waves, Nature Physics 10 (2014) 658-663.

[37] M. Frigo, S. G. Johnson, The design and implementation of FFTW 3, Proc. IEEE 93 (2) (2005) 216-231.

URL http://fftw.org

[38] GNU Project[link]

URL http://gnu.org

[39] P. M. Lushnikov, V. E. Zakharov, On optimal canonical variables in the theory of ideal fluid with free surface, Physica D 203 (1-2) (2005) 9-29.

[40] A. Pushkarev, V. E. Zakharov, Turbulence of capillary waves, Phys. Rev. Lett. 76 (18) (1996) 3320-3323. 